The Astrophysical JouRnal, 544:747-762, 2000 December 1

(C) 2000. The American Astronomical Society. All rights reserved. Printed in U.S.A.

\title{
THE FREQUENCY OF NUCLEAR STAR FORMATION IN SEYFERT 2 GALAXIES
}

\author{
Thaisa Storchi-Bergmann, ${ }^{1}$ Daniel Raimann, ${ }^{2}$ Eduardo L. D. Bica, and Henrique A. Fraquelli ${ }^{2}$ \\ Instituto de Física-UFRGS, CP 15051, CEP 91501-970, Porto Alegre, RS, Brazil; thaisa@if.ufrgs.br, raimann@if.ufrgs.br, bica@if.ufrgs.br, ico@if.ufrgs.br \\ Received 2000 April 10; accepted 2000 July 13
}

\begin{abstract}
We investigate the detectability of starburst signatures in the nuclear spectrum of Seyfert 2 galaxies by constructing spectral models in the wavelength range $\lambda \lambda 3500-4100$, combining the spectrum of a bulge population (of age $\approx 10 \mathrm{Gyr}$ ) with that of younger stellar populations, spanning ages from $\approx 3 \mathrm{Myr}$ to 1 Gyr. The major constraints in the analysis are (1) the continuum ratio $\lambda \lambda 3660 / 4020$, which efficiently discriminates between models combining a bulge spectrum with a stellar population younger than $\approx 50$ Myr and those with older stellar populations; (2) the presence of the Balmer lines H8, H9, and H10 in absorption, which are unambiguous signatures of stellar populations with ages in the range $10 \mathrm{Myr}-1$ Gyr for the relevant metallicities. Their detectability depends both on the age of the young component and on its contribution to the total flux relative to that of the bulge. We also construct models combining the bulge template with a power-law (PL) continuum, which is observed in some Seyfert 2 galaxies in polarized light, contributing with typically $10 \%-40 \%$ of the flux at $4020 \AA$. We conclude that such continuum cannot be distinguished from that of a very young stellar population (age $\leq 10 \mathrm{Myr}$ ), contributing with less than $\approx 0.02 \%$ of the mass of the bulge. The models are compared with nuclear spectra-corresponding to a radius of 200-300 pc at the galaxy-of 20 Seyfert 2 galaxies, in which we specifically look for the signatures above of young- to intermediate-aged stellar populations. We find them in 10 galaxies, thus $50 \%$ of the sample. But only in six cases $(30 \%$ of the sample) can they be attributed to young stars (age < 500 Myr): Mrk 1210, ESO 362-G8, NGC 5135, NGC 5643, NGC 7130, and NGC 7582. In the remaining four cases, the signatures are caused by intermediate-aged stars $(\approx 1$ Gyr). We find a tendency for the young stars to be found more frequently among the late-type Seyfert galaxies, a well-known effect in the nuclei of normal galaxies. This tendency is supported by a comparison between the equivalent widths $(W)$ of absorption lines of the nuclear spectra of the Seyfert 2 galaxies with those of normal galaxies of the same Hubble type. For the late-type galaxies, the $W$ values of the Seyfert galaxies are within the observed range of the normal galaxies, suggesting a similar stellar population. On the other hand, the $W$ values are lower than those of the normal galaxies for seven out of the 11 Seyferts in early-type galaxies.

Subject headings: galaxies: active — galaxies: nuclei — galaxies: Seyfert — galaxies: stellar content
\end{abstract}

\section{INTRODUCTION}

The connection between star formation and nuclear activity in galaxies has been the subject of a number of recent studies. On the theoretical side, models such as those of Perry \& Dyson (1985) and Norman \& Scoville (1988) propose that a nuclear young stellar cluster is the reservoir of fuel for the active galactic nucleus (AGN) in the nucleus.

On the observational side, Terlevich, Diaz, \& Terlevich (1990) have argued that for a number of Seyfert 2 galaxies, circumnuclear starbursts are necessary to explain the strength of the Ca II triplet (at $\lambda \approx 8500 \AA$ ) in absorption. Starbursts also have been proposed as the source of the blue unpolarized continuum observed in Seyfert 2 galaxies (FC2, Tran 1995a, 1995b, 1995c) by Cid Fernandes \& Terlevich $(1992,1995)$. Heckman et al. (1995) have proposed the same on the basis of the IUE spectra of the brightest Seyfert 2 galaxies in the UV. Detailed studies of individual cases have been performed by Heckman et al. (1997), who have shown HST UV images and spectra of the circumnuclear starburst around Mrk 477, and by González Delgado et al. (1998), who have analyzed similar data for the starbursts around

\footnotetext{
${ }^{1}$ Visiting Astronomer at the Cerro Tololo Inter-American Observatory, operated by the Association of Universities for Research in Astronomy, Inc., under contract with the National Science Foundation.

${ }^{2}$ Conselho Nacional de Pesquisas Fellow (Brazil).
}

the Seyfert 2 nuclei of NGC 7130, NGC 5135, and IC 3639. The latter studies have shown the most unambiguous signatures of massive starbursts around these nuclei but have been performed for only four galaxies.

Our main goal in this work is to extend to a larger sample the search of such nuclear starbursts signatures in AGNs. Recent works (e.g., González Delgado, Leitherer, \& Heckman 1999) have pointed out that some of the best spectral features to date of starbursts are found in the blue near-UV spectral region (3400-4200 ̊), the strongest being the higher order Balmer absorption lines (hereafter HOBL). These features seem indeed to be present in the spectra of most starburst galaxies, as can be observed in the sample of Storchi-Bergmann, Kinney, \& Challis (1995). Bica, Alloin, \& Schmitt (1994, hereafter BAS94) also have pointed out the importance of this spectral range for the interpretation of composite stellar populations because of the signatures of the younger components (e.g., Balmer lines and Balmer jump) and the $4000 \AA$ break related to the old- and intermediate-aged $(\approx 1-5 \mathrm{Gyr})$ populations. They studied near-UV/blue spectra of star clusters of all ages and analyzed equivalent widths of a number of features and continuum fluxes as a function of age and metallicity. Figure 1 of BAS94 is particularly illustrative of the variation of the HOBL as a function of age and metallicity.

To look for starbursts in Seyfert galaxies, one has to take into account the contribution of the underlying bulge popu- 
lation. Simulations of starburst spectra superimposed on older stellar populations, for the spectral range at 3700 $9600 \AA$ were carried out by Bica, Alloin, \& Schmidt (1990, hereafter BAS90; see also Schmidt, Alloin, \& Bica 1995). For three relative amounts of mass stocked in star formation events, they combined a bulge spectrum with spectra of star cluster templates of different ages to simulate the evolution of equivalent widths and continuum fluxes. In this study, we construct similar models in the range 3600-4100 $\AA$, which is particularly important for the Seyfert 2 issue. The present models represent an improvement relative to BAS90 in the sense that the templates incorporate the high signal-to-noise ratio $(\mathrm{S} / \mathrm{N})$ near-UV range constructed from the star cluster spectra of BAS94.

We then apply the results of the above simulations to the nuclear spectra of 20 Seyfert 2 galaxies to quantify the contribution of a possible nuclear starburst to the spectrum. The data used here are the nuclear spectra of the 20 Seyfert 2s of the sample of Cid Fernandes, Schmitt, \& StorchiBergmann (1998). Schmitt, Storchi-Bergmann, \& Cid Fernandes (1999) have performed a spectral synthesis using these spectra, but most features used in the synthesis have $\lambda>4000 \AA$. Our goal here is to take a close look at the near-UV region. The aperture used in the extraction of the nuclear spectra $\left(2^{\prime \prime} \times 2^{\prime \prime}\right)$ corresponds at the galaxy to regions within a radius of 200-300 pc from the nucleus, which are of the order of the nuclear starburst sizes investigated in detail by González Delgado et al. (1998).

The second goal of this study is to compare the results obtained for the Seyfert galaxies with those for normal galaxies of the same Hubble type. Cid Fernandes et al. (1998) have shown that in many Seyfert $2 \mathrm{~s}$, the blue nuclear continuum seems to be absent when one takes into account the surrounding stellar population. In other words, they have found that the nuclear stellar population is varied and usually differs from that of an elliptical galaxy, as adopted in early works. The blue continuum artificially appears when one subtracts an elliptical galaxy template from the nuclear spectrum of a galaxy containing some contribution of younger stars (Storchi-Bergmann, Cid Fernandes, \& Schmitt 1998). The spectral syntheses of Schmitt et al. (1999) have further shown that the main difference between the nuclear stellar population of Seyfert $2 \mathrm{~s}$ and that of an elliptical galaxy is an excess contribution of a $\approx 100 \mathrm{Myr}$ stellar population. These findings point to $\mathrm{FC} 2$ as being caused by young stars in several cases, but another important question is whether the contribution of young stars in Seyfert nuclei is larger than that in normal galaxies of the same Hubble type. Since Seyfert nuclei as a rule occur in spiral galaxies, some contribution of young stars is expected. To access this aspect, it is necessary to compare the nuclear stellar population characteristics of the Seyfert galaxies with those of normal galaxies. We thus assume the spectral study of Bica \& Alloin (1987) as representative of the results obtained for normal galaxies to make such a comparison.

The paper is organized as follows. In $\S 2$, we discuss the near-UV features and adopted templates; in $\S 3$, we present the spectral models; in $\S 4$, we compare the nuclear Seyfert 2 spectra with the models; in $\S 5$, we compare our results with those of similar studies; in $\S 6$, we discuss the degeneracy problem between a featureless power law and a very young starburst continuum; in $\S 7$, we compare the results for the Seyfert galaxies with those for normal galaxies of the same Hubble type and, finally, in $\S 8$, we present our conclusions.

\section{NEAR-ULTRAVIOLET FEATURES AND ADOPTED TEMPLATES}

The spectral features in the near-UV for a red (old/ intermediate age) stellar population are markedly different from those of younger stellar populations. This can be observed in Figure 1 (see also Fig. 2 of BAS94), where we show the stellar population templates used in this blueviolet study. The star cluster and early-type galaxy bulge (E2E5 of BAS94) templates used here incorporate observations from near-UV and visible (Bica \& Alloin 1986, 1987) domains. The templates also extend further to the UV and near-IR domains, which can be used as additional constraints in the analysis. A detailed description of these templates is given by Bica and collaborators in $\S 4.1$ of Leitherer et al. (1996). To represent a 3 Myr stellar population, we adopt the integrated spectrum of 30 Doradus (BAS94, Bica \& Alloin 1986).

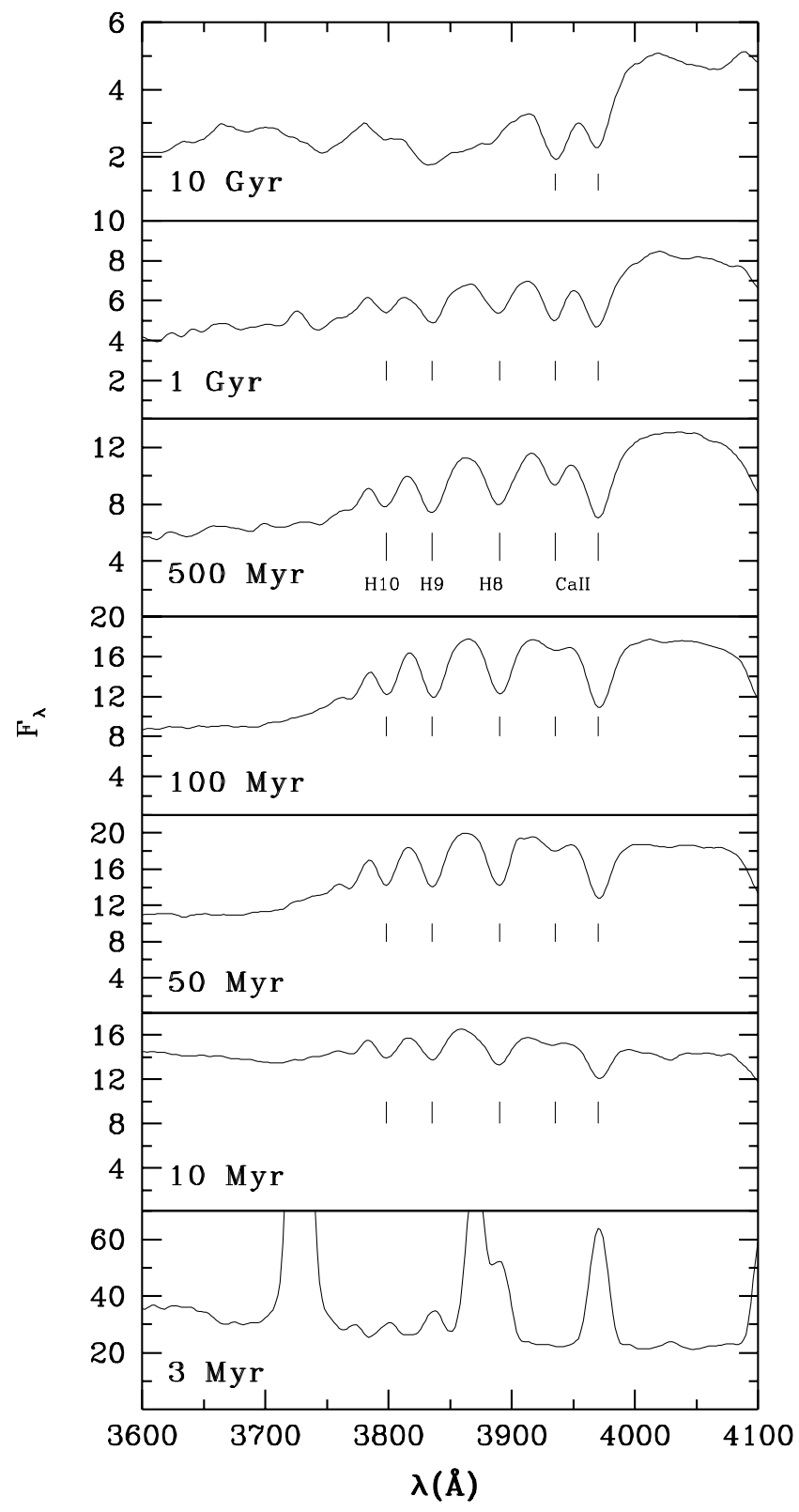

FIG. 1.-Stellar population templates, corresponding to ages from 3 Myr to $10 \mathrm{Gyr}$. Main absorption features are identified. 
In the old stellar population template, typical of elliptical galaxies or of the bulge of spirals, the main absorption features are the $\mathrm{Ca}$ II $\mathrm{K}(\lambda 3933)$ and $\mathrm{H}(\lambda 3970)$ lines and a blend of absorptions caused by $\mathrm{CN}, \mathrm{Mg}$ I, $\mathrm{Si}$ I, and $\mathrm{Fe}$ I. For the younger stellar populations, with ages between $10 \mathrm{Myr}$ and $1 \mathrm{Gyr}$, the main features are, apart from the $\mathrm{Ca}$ II $\mathrm{K}$ and $\mathrm{H}$ lines with varying strengths, the high-order Balmer lines in absorption, in particular $\mathrm{H} \delta(\lambda 4102), \mathrm{H} \epsilon(\lambda 3970), \mathrm{H} 8(\lambda 3889)$,

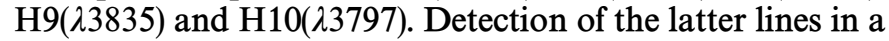
spectrum is thus a strong signature of the presence of a blue (young) stellar population, except when the burst is younger than $5 \mathrm{Myr}$, in which case these absorptions are filled by emission.

\section{MODELS}

Following BAS90, we have used the template bulge spectra in combination with varying proportions of the younger templates to build simple model spectra representative of composite populations. We have combined the bulge component with $0.1 \%, 1 \%$, and $10 \%$ mass contributions locked in the younger components to construct the models shown in Figures 2, 3, and 4. The adopted mass-tolight ratios in $V$ (BAS90) for the templates representing bursts of different ages, together with the relative luminosities at $4020 \AA L_{\lambda 4020}$ for bursts of equal masses, are listed in Table 1. This table can be used to find the contribution in flux at $4020 \AA$ corresponding to a given mass proportion. For example, to construct the model combining the bulge with $0.1 \%$ in mass of the $10 \mathrm{Myr}$ population, we have multiplied $L_{\lambda 4020}$ of the $10 \mathrm{Myr}$ template by $0.1 \%$ to obtain 3.354 , which is the normalization factor of the template at $4020 \AA$. Subsequently, it is added to the bulge template normalized to 1 at this same wavelength. Notice that for equal masses, the $10 \mathrm{Myr}$ cluster $L_{\lambda 4020}$ value is higher than that of the $3 \mathrm{Myr}$ cluster embedded in the $\mathrm{H}$ II region because of the appearance of supergiants.

By examining the characteristic features of the young stars in Figures 2, 3, and 4, in particular the absorption lines $\mathrm{H} 8, \mathrm{H}$, and $\mathrm{H} 10$ (the HOBL) - which are the ones less affected by emission in Seyfert galaxies (see below) - it can be concluded that (1) if the starburst is very young, with ages of a few Myr, the HOBL are filled by emission - in this case, the $\mathrm{H}$ II region emission spectrum may be used as constraint; (2) for a small starburst, so that its mass is $\approx 0.1 \%$ of that of the bulge, the HOBL can be detected for young starbursts with ages from 10 to $50 \mathrm{Myr}$; (3) for a starburst corresponding to $1 \%$ of the mass of the old stars, the above features can be observed for ages from 10 to 500 Myr; (4) for a stronger starburst, corresponding to $10 \%$ of the mass of the bulge, the features are observed for ages from $10 \mathrm{Myr}$ up to $1 \mathrm{Gyr}$.

TABLE 1

Template Ages, Mass-to-Light Ratios in $V$ AND Relative Luminosity AT $4020 \AA$

\begin{tabular}{clr}
\hline \hline Age & $M / L_{V}$ & $L_{\lambda 4020}$ \\
\hline $3 \mathrm{Myr} \ldots \ldots \ldots \ldots \ldots$ & 0.030 & 1134.0 \\
$10 \mathrm{Myr} \ldots \ldots \ldots \ldots$ & 0.007 & 3354.1 \\
$50 \mathrm{Myr} \ldots \ldots \ldots \ldots$ & 0.054 & 504.4 \\
$100 \mathrm{Myr} \ldots \ldots \ldots \ldots$ & 0.260 & 97.1 \\
$500 \mathrm{Myr} \ldots \ldots \ldots \ldots$ & 0.310 & 63.9 \\
$1 \mathrm{Gyr} \ldots \ldots \ldots \ldots \ldots$ & 2.19 & 6.8 \\
$10 \mathrm{Gyr} \ldots \ldots \ldots \ldots$ & 8.6 & 1.0 \\
\hline
\end{tabular}

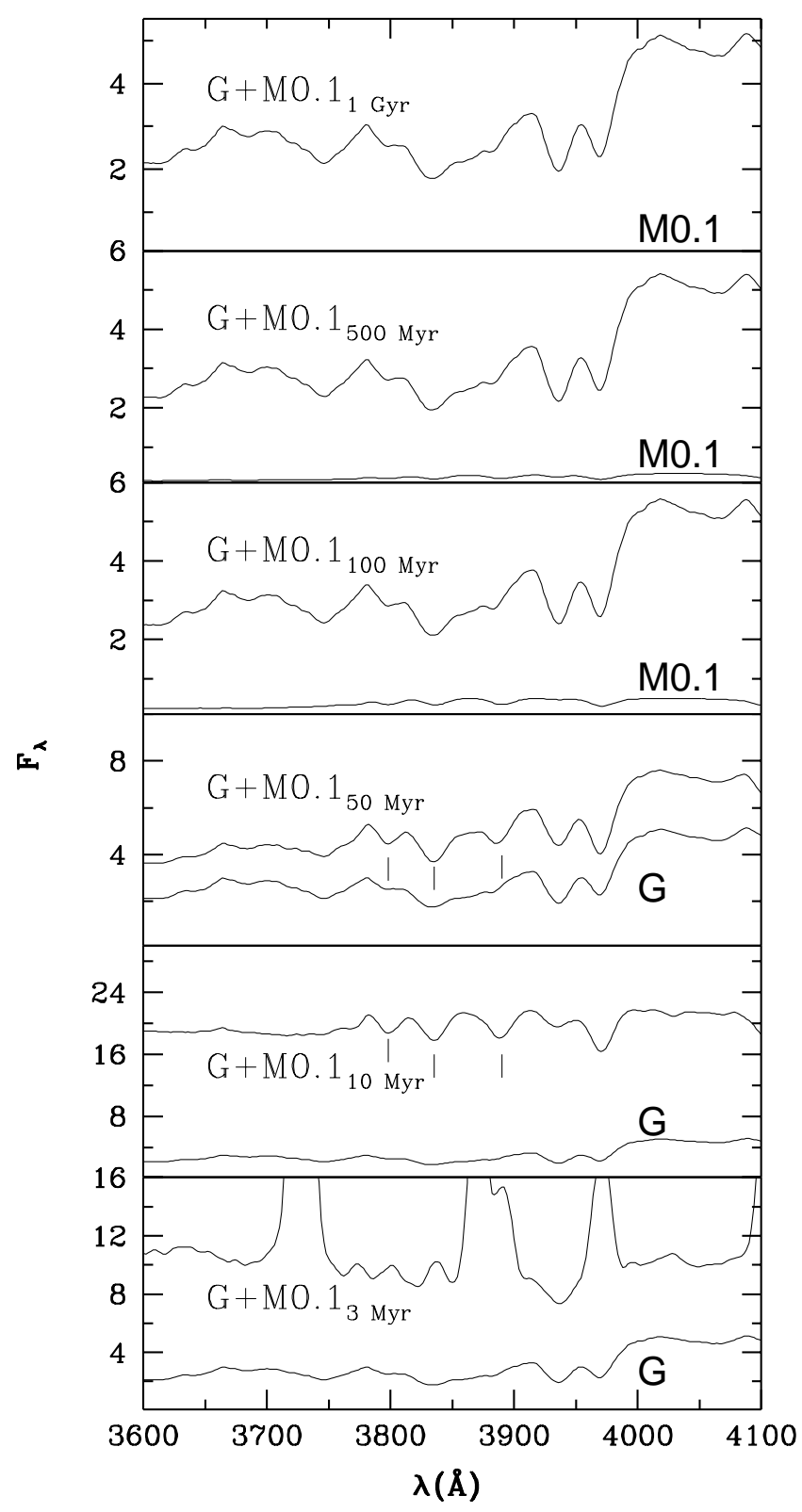

FIG. 2.-Top of each panel: Composite spectra constructed by combining a bulge template $(\mathrm{G})$ plus $0.1 \%$ in mass $(\mathrm{M})$ of bursts with ages from 3 Myr to 1 Gyr. Bottom of each panel: In the three lower panels, contribution of the bulge component, and in the three upper panels, contribution of the young component.

To quantitatively characterize stellar populations, BAS94 have proposed the use of a few continuum points whereby a continuum is traced (connecting these points using straight lines) to measure the equivalent widths (hereafter represented by $W$ ) of a number of features. However, in Seyfert galaxies, many of these features are filled by emission lines and cannot be used. By comparing the above stellar population templates with typical Seyfert spectra, we conclude that the windows that are usually free from emission lines are $\lambda \lambda 3810-3822$ (centered on a continuum point), $\lambda \lambda 3822-3858$ (centered on H9), and $\lambda 23908-3952$ (centered on the Ca II K line). We thus have followed the method of BAS90 to construct the continuum and have measured the $W$ 's within the three windows above, hereafter identified as $W_{C}, W_{\mathrm{H} 9}$, and $W_{\text {Ca I K }}$, respectively.Figure 5 illustrates the continuum and 


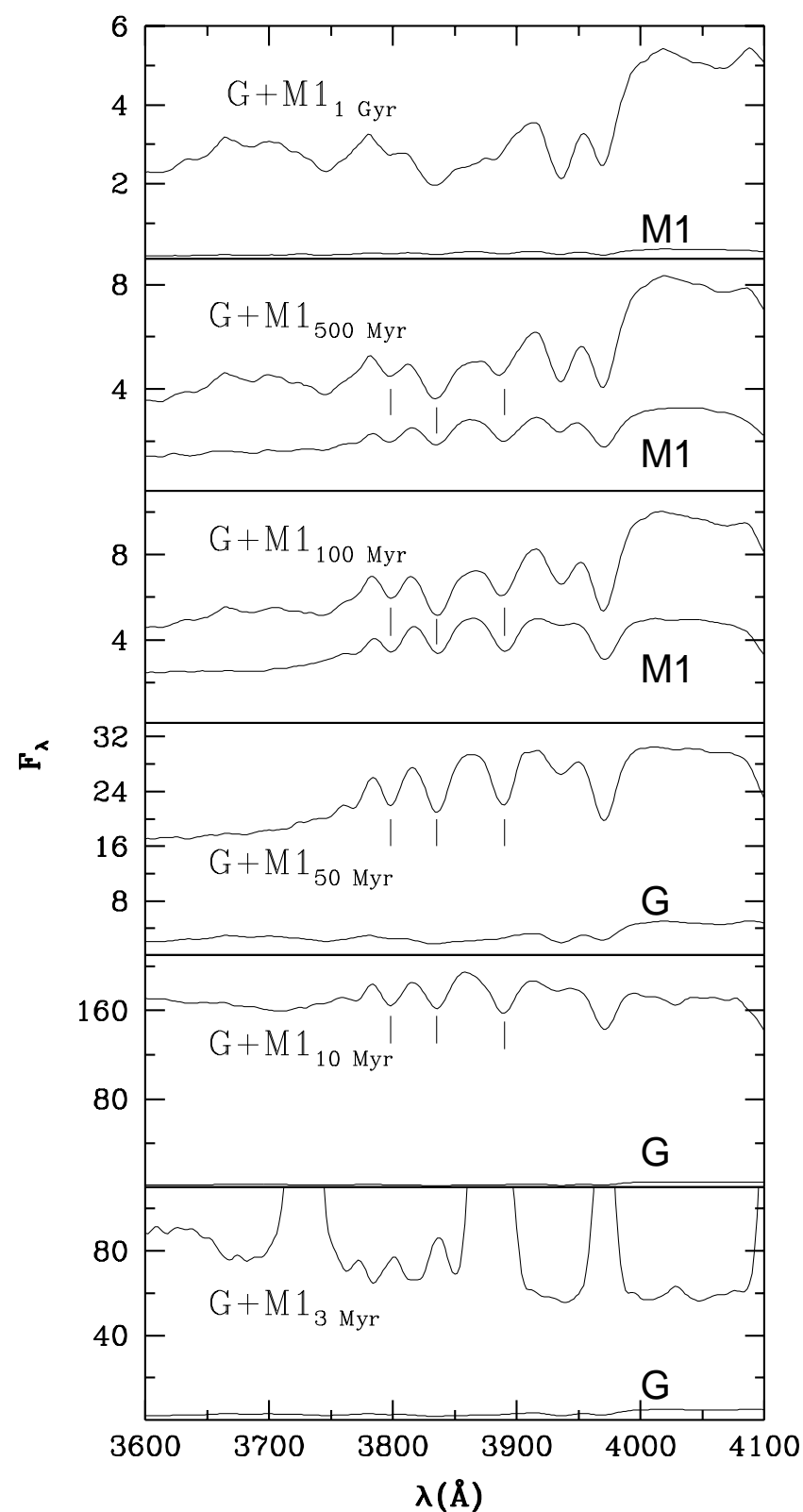

FIG. 3.-Top of each panel: Composite spectrum constructed by combining a bulge template $(\mathrm{G})$ plus $1 \%$ in mass $(\mathrm{M})$ of bursts with ages from 3 Myr to 1 Gyr. Bottom of each panel: In the three lower panels, contribution of the bulge component, and in the three upper panels, contribution of the young component.

windows used for three Seyfert 2 galaxies with distinct near-UV spectra.

The near-UV $W$ 's and continuum ratio $\lambda \lambda 3660 / 4020$ (hereafter CR) were measured for the models. We show in Figure $6 \mathrm{CR}, W_{C}$, and $W_{\mathrm{Ca} \Pi \mathrm{K}}$ as a function of the young component's age for the three proportions in mass above. It can be concluded that $\mathrm{CR}\left(\mathrm{F}_{3660} / \mathrm{F}_{4020}\right)$ is a powerful star formation tracer for very young stellar populations, varying from 1.4 for a $3 \mathrm{Myr}$ stellar population down to 0.6 for 50 Myr or older. $W_{C}$ and $W_{\text {Ca I K }}$ are better age indicators for older bursts but can trace stellar populations of all ages, from $3 \mathrm{Myr}$ to $1 \mathrm{Gyr}$. We recall that blue-violet metal lines in composite spectra are age indicators because of the dilution effects caused by hot main-sequence stars (Bica \& Alloin 1986). Regarding H9, we conclude that its profile,

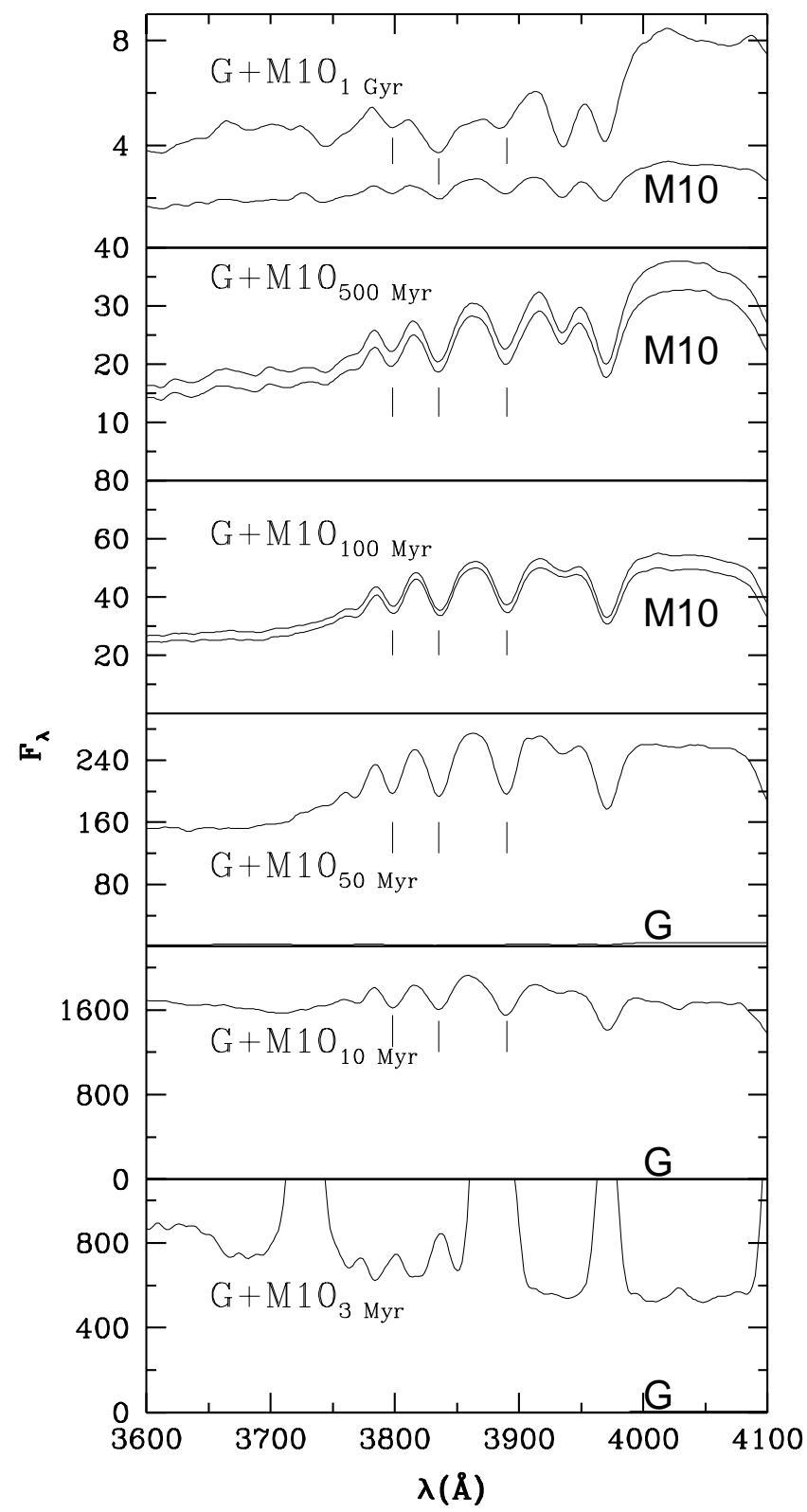

FIG. 4.-Top of each panel: Composite spectra constructed by combining a bulge template $(\mathrm{G})$ plus $10 \%$ in mass $(\mathrm{M})$ of bursts with ages from 3 Myr to 1 Gyr. Bottom of each panel: In the three lower panels, contribution of the bulge component, and in the three upper panels, contribution of the young component.

together with those of the other HOBL are better age indicators than $W_{\mathrm{H} 9}$.

A number of Seyfert 2 galaxies present a polarized blue continuum with a power-law spectrum (Tran 1995a, 1995b, 1995c; Storchi-Bergmann et al. 1998). Thus we also have constructed a second set of models combining the bulge template and a power law $F_{v} \propto v^{-1.5}$ (hereafter PL) typical of the polarized continuum found by Tran $(1995 \mathrm{a}, 1995 \mathrm{~b}$, 1995c). As the mass proportions used above have no meaning for the PL, we have used combinations with varying proportions of the PL in flux at $4020 \AA$.

The CR, $W_{C}$, and $W_{\mathrm{Ca} \text { I K }}$ measured for the models combining the bulge template with the PL are plotted in Figure 7 as a function of the PL percent contribution in flux at $4020 \AA$. As a comparison, we also plot the $W$ values from 


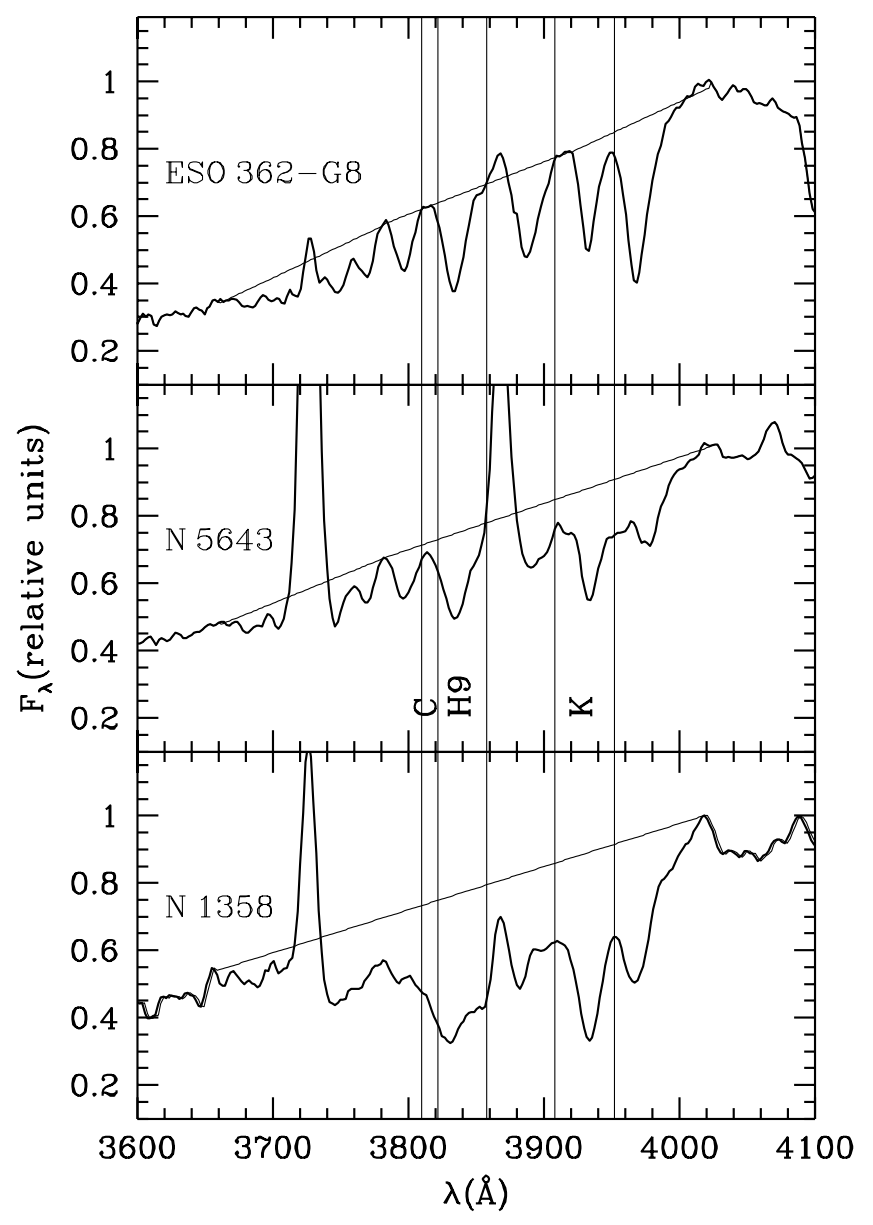

FIG. 5.-Illustration of the continuum and windows (vertical lines) used in the measurements of the equivalent widths $W_{C}, W_{\mathrm{H} 9}$, and $W_{\mathrm{Ca} \mathrm{II}}$ for three Seyfert type 2 galaxies of the sample.

models combining a bulge with the same flux contribution from a $10 \mathrm{Myr}$ stellar population and a $100 \mathrm{Myr}$ population. Combination with older populations produces similar CR values as the latter, as can be seen in Figure 6. Figure 7 shows that $\mathrm{CR}$ is the most powerful discriminator between a PL and bursts of $\approx 100 \mathrm{Myr}$ or older. On the other hand, it also shows that a PL is hardly distinguishable from a $10 \mathrm{Myr}$ burst on the basis of the CR and $W$ values, a problem that has been pointed out already by several authors and by ourselves in Schmitt et al. (1999). We discuss this problem further in $\S 6$.

\section{COMPARISON WITH SEYFERT GALAXIES}

The Seyfert sample used in this work is the same as that of Schmitt et al. (1999, hereafter SSC99), except for one radio galaxy (which had a low $\mathrm{S} / \mathrm{N}$ spectrum in the nearUV), and consists of the 20 Seyfert type 2 galaxies and three radio galaxies from the larger sample of Cid Fernandes et al. (1998). As in SSC99, we use here only the nuclear spectra, extracted using windows of $2^{\prime \prime} \times 2^{\prime \prime}$, which correspond at the galaxy to regions of typical diameters of a few hundred parsecs.

These spectra are plotted in Figures 8, 9, 10, 11, and 12, separated according to the Hubble types. Here we have made use of the updated morphological classifications of our sample of Seyfert 2 galaxies in the Malkan, Gorjian, \&
Tam (1998) HST optical imaging survey because of the better spatial resolution and dynamical range of their images as compared with those upon which the previous classifications (de Vaucouleurs et al. 1991, hereafter RC3) are based. We found an interesting result: out of the 20 galaxies in our sample, 10 have new classifications when compared with those of $\mathrm{RC} 3$, always to later Hubble types, as is shown in Table 2. In particular, two of the galaxies with the most obvious signatures of recent star formation, NGC 5135 and NGC 7130 (González Delgado et al. 1998), previously classified as $\mathrm{Sab}$ and $\mathrm{Sa}$, respectively, have been reclassified as Sc and Sd by Malkan et al. (1998).

The galaxies CR, $W$ values, and scales $(\mathrm{pc} / \mathrm{arcsec})$ are listed in Table 3. Typical errors are $\sim 0.01$ in CR and $\leq 1 \AA$ in the $W$ values. In Cid Fernandes et al. (1998), we noticed that the nuclear spectra of these galaxies were in most cases redder than the extranuclear neighboring spectra, a result we attributed to reddening. Indeed, in the spectral syntheses performed by SSC99, they usually found significant reddenings $(0.1 \leq E(B-V) \leq 0.60)$. Thus we have corrected the nuclear spectra by the reddening (Seaton 1979) found by SSC99 before tracing the continuum and measuring the near-UV, $W$ values, and CR. Notice, however, that the reddening corrections are not critical for $\mathrm{CR}$ because of the proximity of the continuum wavelengths $\lambda \lambda 3660$ and 4020 .

We now compare the near-UV nuclear spectra of our sample with the synthetic spectra derived from the CR and $W$ values listed in Table 3 using the model values from Figures 6 and 7.

As the templates have lower spectral resolution $(\approx 15 \AA)$ than the Seyfert spectra $(\approx 5 \AA)$, we present here the models compared with the Seyfert spectra smoothed to better match the template resolution. The smoothing may cause loss of information, particularly when there are faint emission lines superimposed on the HOBL. Therefore, we have always checked for these emission lines at full resolution and have not included in the fit the absorption features affected by line emission. The most frequent case is the contamination of the $\mathrm{Ca}$ II $\mathrm{H}+\mathrm{H} \epsilon$ absorption by $\mathrm{Ne}$ III $\lambda 3968+\mathrm{H} \epsilon$ emission.

Our goal with these comparisons is not a perfect match of the nuclear spectrum but to identify the unambiguous signatures of the different components to the nuclear spectrum, looking in particular for the characteristic star formation features HOBL ( $\mathrm{H} 8, \mathrm{H} 9$, and $\mathrm{H} 10$ in absorption) using the simple models as a guide. With this approach, we also check if these simple models are a good representation of the nuclear stellar population. In applying the models of Figures 6 and 7, it can be noted that the CRs and $W$ values

TABLE 2

Previous and New Classifications

\begin{tabular}{ccc}
\hline \hline Galaxy & RC3 & Malkan et al. \\
\hline Mrk $607 \ldots \ldots \ldots \ldots \ldots \ldots$ & Sa & Sb \\
NGC $1386 \ldots \ldots \ldots \ldots \ldots$ & SB0 & Sbc \\
CGCG $420-015 \ldots \ldots \ldots$ & $?$ & Sa \\
ESO $362-G 8 \ldots \ldots \ldots \ldots$. & S0 & Sa \\
Mrk $1210 \ldots \ldots \ldots \ldots \ldots$ & S $?$ & Sa \\
IRAS $11215 \ldots 2806 \ldots \ldots$ & $?$ & S0 \\
MCG $-05-27-013 \ldots \ldots$ & SBa & Sb \\
NGC $5135 \ldots \ldots \ldots \ldots \ldots$ & SBab & Sc \\
NGC $6300 \ldots \ldots \ldots \ldots \ldots$ & SBb & Sd \\
NGC $7130 \ldots \ldots \ldots \ldots \ldots$ & Sa & Sd \\
\hline
\end{tabular}



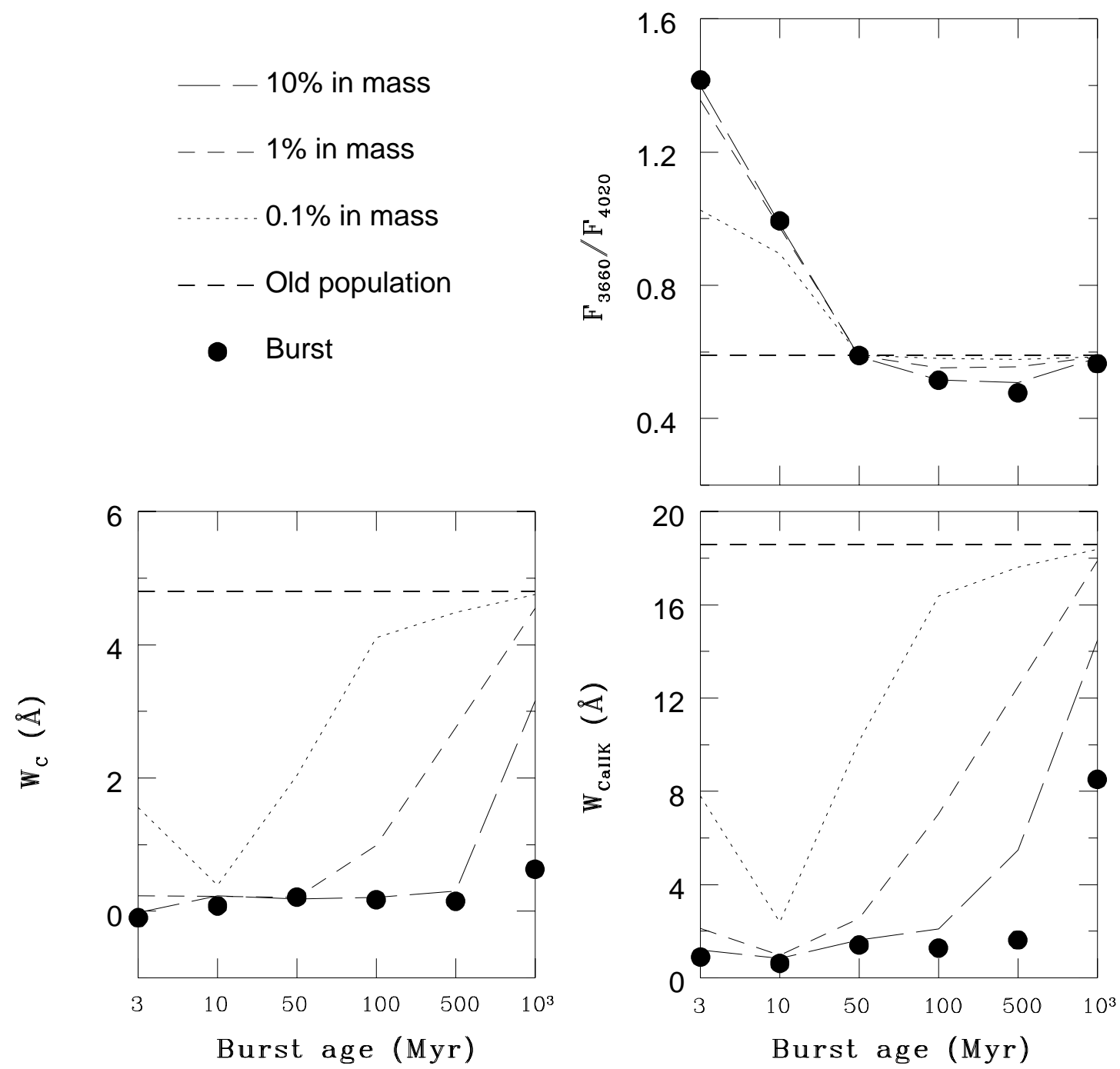

FIG. 6.-Continuum ratio $\mathrm{CR}=\mathrm{F}_{3660} / \mathrm{F}_{4020}$ and equivalent widths $W_{C}$ and $W_{\mathrm{Ca} \mathrm{II}}$ for synthetic spectra constructed combining a bulge template and bursts of varying ages contributing with zero (heavy dashed line), $0.1 \%$ (dotted line), $1 \%$ (dashed line), $10 \%$ (long-dashed line), and $100 \%$ ( filled circles) in mass.

are compatible in several cases with more than one model. In these cases, the adopted model is the one that gives the best fit to the overall spectrum. The quality of the fit is inspected in regions free from emission lines, and the best fit is the one that gives the smaller residuals between the observed and model spectra in these regions.

As pointed out above, it is not possible to distinguish a PL continuum from that of a template of age $10 \mathrm{Myr}$ or younger for flux contributions smaller than $40 \%$ at $4020 \AA$. When such a continuum is needed, we will call it PL/YS, meaning power law or young stars. The nature of this component is discussed further in $\S 6$.

If the Seyfert 2 has strong Balmer emission lines, the Balmer continuum in emission may be important in the near-UV region, diluting the $W$ values calculated above. From the emission-line fluxes, we found that the Balmer continuum only contributes significantly (with more than $5 \%$ to the flux) for $\lambda<3646 \AA$, for the galaxies IC 1816 , MCG -05-27-013, Mrk 348, Mrk 573, and Mrk 1210. In these cases, we have considered also the contribution of the Balmer continuum, calculated as in Osterbrock (1989), and normalized according to the fluxes of the Balmer emission lines.

\subsection{Elliptical Galaxies}

In order to verify the applicability of the templates as a basis for synthesizing the Seyfert spectra, which were observed with the CTIO $4 \mathrm{~m}$ Blanco telescope (Cid Fernandes et al. 1998), we have first applied the models to the normal elliptical galaxy IC 4889, observed with the same telescope, as a test to the method. In addition, we apply the models to three radio elliptical galaxies. The spectra and models are illustrated in Figure 13.

\subsubsection{The Normal Elliptical IC 4889}

The near-UV $W$ values and CR agree very well with those of the bulge template and indicate no need of bluer components, as expected. Figure $13 a$ illustrates the observed spectrum as compared with the bulge template, showing a very good match of the observed spectrum to the template, indicating that we can use the templates constructed from the cluster spectra to synthesize the Seyfert nuclear spectra. Small differences are apparent in the Ca II $\mathrm{K}$ and $\mathrm{H}$ lines, which are deeper in the galaxy spectrum, even though the $W$ values are the same as in the bulge template. We attribute this effect to a residual difference in 

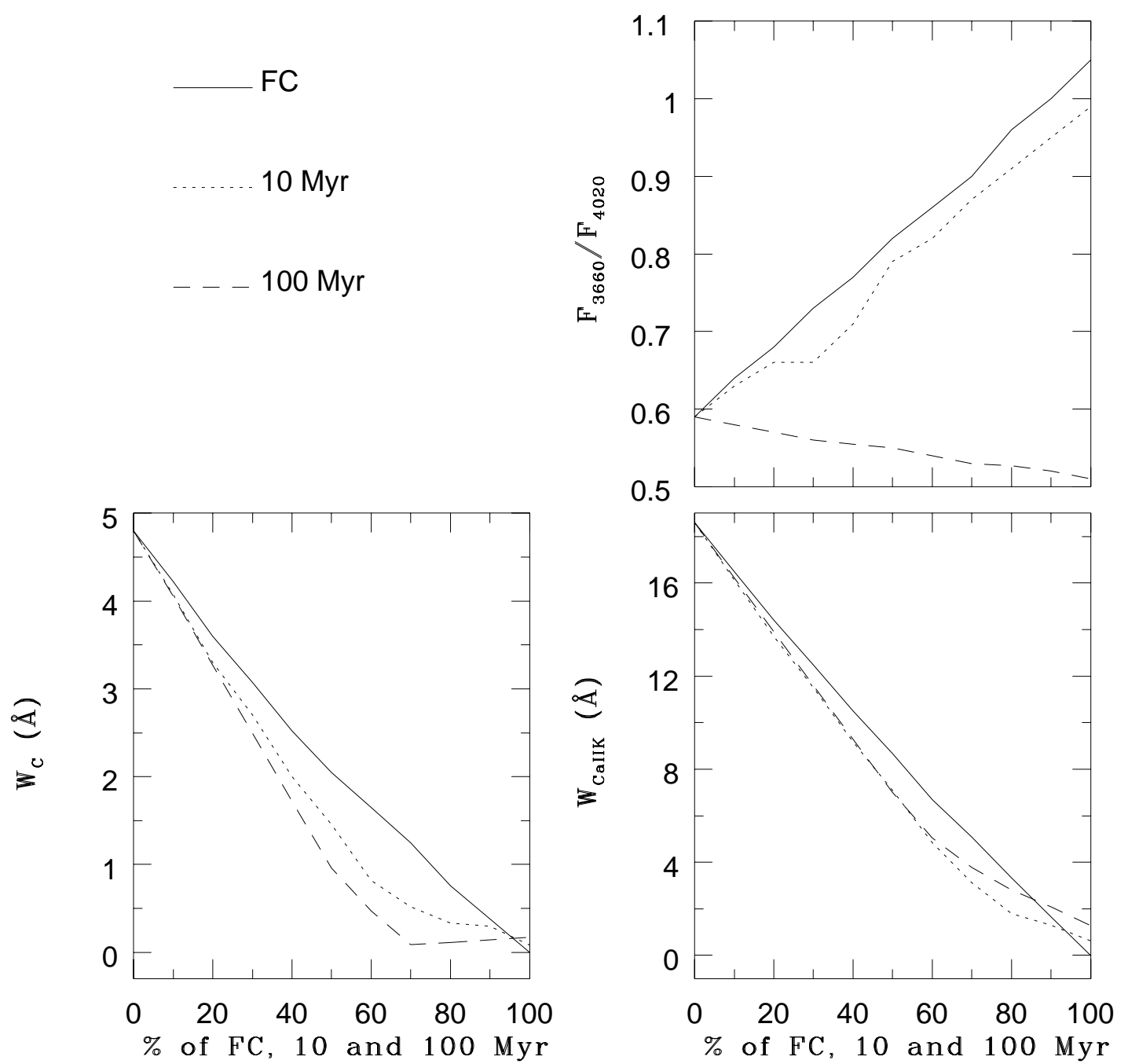

FIG. 7. - Continuum ratio $\mathrm{CR}=\mathrm{F}_{3660} / \mathrm{F}_{4020}$ and equivalent widths $W_{C}$ and $W_{\text {Ca II K }}$ for synthetic spectra constructed combining a bulge template and increasing fractions (in flux at $4020 \AA$ ) of a PL (solid line), a $10 \mathrm{Myr}$ (dotted line), and a $100 \mathrm{Myr}$ (dashed line) burst template.

spectral resolution between our spectrum and the bulge template.

\subsection{2. $3 C 33$}

This is a radio galaxy with near-UV $W$ values smaller than those of the bulge template, indicating the presence of some contribution from a blue component. The best fit for the spectrum is obtained with the combination of a bulge plus $10 \%$ in mass of a population of $1 \mathrm{Gyr}$, although the nuclear spectrum of the galaxy is still somewhat bluer for $\lambda<3660 \AA$ A. An improved fit to the blue end of the spectrum is obtained by adding a $5 \%$ contribution in flux at $\lambda 4020$ of the PL/YS component. The HOBL are at the detection limit (Fig. 13b). Ne III $\lambda 3968+\mathrm{H} \epsilon$ emission can be observed filling the $\mathrm{Ca}$ II $\mathrm{H}+\mathrm{H} \epsilon$ absorption.

\subsubsection{PKS $0349-27$}

This radio galaxy has a small dilution of the $W$ values when compared with the elliptical template values. The overall spectral distribution is best reproduced by the combination of the bulge with $10 \%$ flux contribution of the PL/YS component at $4020 \AA$ (Fig. 13c). The difference in the depth of the Ca II $\mathrm{K}$ line is similar to that observed in IC 4889 and thus caused by the better spectral resolution of the radio galaxy spectrum relative to the template. It is not possible to identify the HOBL. Ne III $\lambda 3968+\mathrm{H} \epsilon$ emission is filling the $\mathrm{Ca} I \mathrm{H}+\mathrm{H} \epsilon$ absorption.

\subsubsection{PKS $0634-20$}

Here the case is similar to the one above, with a somewhat larger dilution in the continuum window and somewhat larger $\mathrm{CR}$. The $\mathrm{CR}$ value indicates a $15 \% \mathrm{PL} / \mathrm{YS}$ contribution, in agreement with the $W$ values and overall spectral distribution (Fig. 13d). The same remark above about the $\mathrm{Ca}$ II $\mathrm{K}$ line applies here. It is not possible to identify the HOBL. There is contamination of the Ca II $\mathrm{H}+\mathrm{H} \epsilon$ absorption by $\mathrm{Ne}$ III $\lambda 3968+\mathrm{H} \epsilon$ emission.

In summary, the near-UV spectrum of the three radio galaxies, when compared with normal ellipticals, shows a systematic need of a small $(10 \%-20 \%$ in flux at $4020 \AA)$ contribution of a blue component. For the two PKS sources, this component is well reproduced by a PL/YS continuum. For 3C 33, it is necessary to also add the contribution of a $1 \mathrm{Gyr}$ stellar population with $\approx 10 \%$ of the bulge component mass. A possible interpretation for such large intermediate-aged contributions would be the cannibalism of a small spiral galaxy or Magellan irregular, as is the case, for example, of the nearby radio galaxy Centaurus A (e.g., Storchi-Bergmann et al. 1997). 


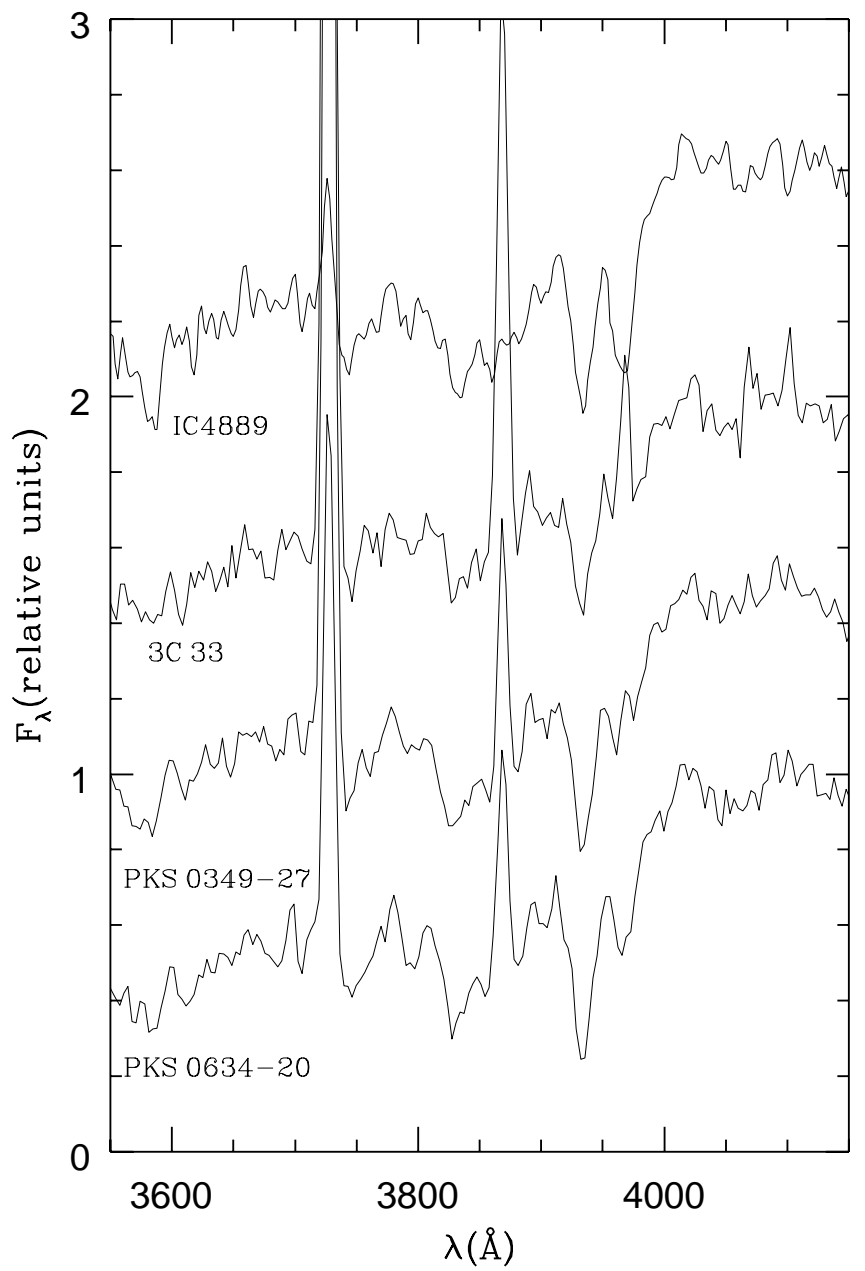

FIG. 8.- Spectra of the elliptical and three radio galaxies of the sample normalized at $4020 \AA$ and shifted for clarity.

\section{2. $S 0$ and $S 0 a$ \\ 4.2.1. $N G C 1358$}

In this galaxy, the near-UV nuclear features and spectral distribution are well represented by the bulge template alone (Fig. 14a). There is some contamination of the Ca II $\mathrm{H}+\mathrm{H} \epsilon$ absorption by Ne III $\lambda 3968+\mathrm{H} \epsilon$ emission.

\subsubsection{NGC 3081}

The CR indicates the presence of a PL/YS component contributing with $\approx 25 \%$ of the flux at $4020 \AA$. The nearUV $W$ values and overall spectrum are also well reproduced by the above combination (Fig. 14b). HOBL are not detected. Ne III $\lambda 3968+\mathrm{H} \epsilon$ emission can be observed filling the $\mathrm{Ca}$ II $\mathrm{H}+\mathrm{H} \epsilon$ absorption.

\subsubsection{Markarian 348}

CR is 0.72 for this galaxy, thus indicating the presence of a PL/YS component contributing with $30 \%$ in flux at 4020 $\AA$ and in approximate agreement with the $W$ values. Figure $14 c$ also shows that the overall spectrum is well reproduced by this combination, taking into account that there is some emission in $\mathrm{H} 9$ and probably also in H10, making it impossible to detect the HOBL in absorption, if any. Ne III $\lambda 3968+\mathrm{H} \epsilon$ emission completely dominates the $\mathrm{Ca}$ II $\mathrm{H}+\mathrm{H} \epsilon$ absorption.

\subsubsection{Markarian 573}

H9 is filled by emission. The CR indicates the presence of a PL/YS component contributing with $\approx 20 \%$ in flux at $4020 \AA$. The other two $W$ values and overall continuum are also well reproduced by this combination (Fig. 14d). The small discrepancy in the $\mathrm{Ca}$ II $\mathrm{K}$ line profile is explained as for the radio galaxies above. HOBL are not detected. Ne III $\lambda 3968+\mathrm{H} \epsilon$ emission completely dominates the $\mathrm{Ca}$ II $\mathrm{H}+\mathrm{H} \epsilon$ absorption.

TABLE 3

Near-ultraviolet Measurements and Scale of the Sample

\begin{tabular}{|c|c|c|c|c|c|}
\hline Name & $\lambda \lambda 3660 / 4020$ & $\begin{array}{l}W_{C} \\
(\AA)\end{array}$ & $\begin{array}{l}W_{\mathrm{H} 9} \\
(\AA)\end{array}$ & $\underset{(\AA)}{W_{\mathrm{Ca}_{\text {II }}} \mathrm{K}}$ & $\begin{array}{c}\text { Scale } \\
\text { (pc/arcsec) }\end{array}$ \\
\hline NGC $1358 \ldots \ldots \ldots \ldots$ & 0.51 & 4.5 & 18.5 & 18.6 & 257 \\
\hline NGC $1386 \ldots \ldots \ldots \ldots$ & 0.52 & 2.5 & 12.9 & 14.0 & 48 \\
\hline NGC $3081 \ldots \ldots \ldots \ldots$ & 0.69 & 3.5 & 12.8 & 14.0 & 140 \\
\hline NGC $5135 \ldots \ldots \ldots \ldots \ldots$ & 0.74 & 0.4 & 4.0 & 2.7 & 256 \\
\hline NGC $5643 \ldots \ldots \ldots \ldots$ & 0.55 & 0.8 & 7.4 & 9.5 & 69 \\
\hline NGC $6300 \ldots \ldots \ldots \ldots$ & 0.54 & 3.0 & 12.9 & 15.8 & 63 \\
\hline NGC $6890 \ldots \ldots \ldots \ldots \ldots$ & 0.72 & 3.0 & 10.1 & 13.7 & 159 \\
\hline NGC $7130 \ldots \ldots \ldots \ldots$ & 0.93 & 0.5 & 3.1 & 3.3 & 314 \\
\hline NGC $7582 \ldots \ldots \ldots \ldots$ & 0.62 & 0.7 & 4.1 & 3.7 & 100 \\
\hline Mrk $348 \ldots \ldots \ldots \ldots \ldots \ldots$ & 0.72 & 2.4 & 8.6 & 13.0 & 302 \\
\hline Mrk $573 \ldots \ldots \ldots \ldots \ldots$ & 0.70 & 3.7 & 11.8 & 15.1 & 334 \\
\hline Mrk $607 \ldots \ldots \ldots \ldots \ldots$ & 0.63 & 2.9 & 12.8 & 14.3 & 176 \\
\hline Mrk $1210 \ldots \ldots$. & 0.83 & 1.6 & $\mathrm{em}^{\mathrm{a}}$ & 9.5 & 253 \\
\hline CGCG 420-015 ......... & 0.57 & 2.6 & 9.8 & 13.8 & 570 \\
\hline IC $1816 \ldots \ldots \ldots \ldots \ldots$ & 0.66 & 2.2 & 6.9 & 12.8 & 328 \\
\hline IRAS $11215-2806 \ldots \ldots$ & 0.55 & 1.4 & 7.4 & 11.8 & 262 \\
\hline MCG $-05-27-013 \ldots \ldots$ & 0.67 & 2.9 & 11.6 & 15.1 & 470 \\
\hline Fairall $316 \ldots \ldots \ldots \ldots$ & 0.57 & 4.6 & 17.9 & 18.2 & 308 \\
\hline ESO $417-G 6 \ldots \ldots \ldots \ldots$ & 0.59 & 3.5 & 13.3 & 15.2 & 310 \\
\hline ESO $362-G 8 \ldots \ldots \ldots \ldots$ & 0.52 & 0.1 & 7.4 & 5.8 & 298 \\
\hline 3C $33 \ldots \ldots \ldots \ldots \ldots$ & 0.60 & 2.4 & 12.9 & 15.2 & 1114 \\
\hline PKS P0349-27........... & 0.60 & 4.4 & 16.1 & 18.5 & 1240 \\
\hline PKS P0634-20........... & 0.66 & 3.4 & 16.2 & 17.9 & 1055 \\
\hline
\end{tabular}

${ }^{a}$ Observed in emission. 


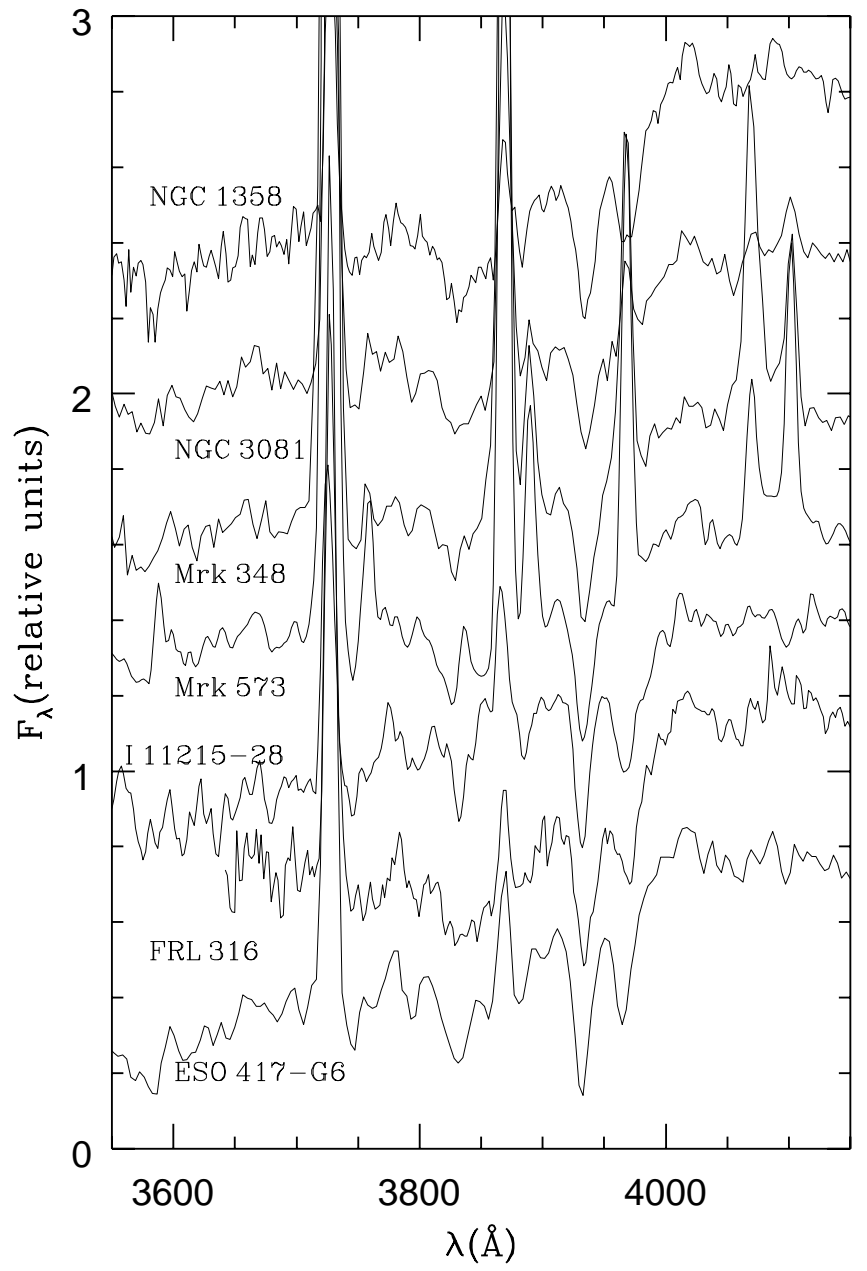

FIG. 9.- Spectra of the seven S0 Seyfert type 2 galaxies of the sample normalized at $4020 \AA$ and shifted for clarity.

\subsubsection{IRAS $11215-2806$}

This galaxy shows significant dilution in the near-UV $W$ values, with the $\mathrm{Ca}$ II $\mathrm{K}$ line profile better reproduced by a combination of a bulge plus $20 \%$ mass contribution of a 1 Gyr component - an additional model we had to construct increasing the contribution of the intermediate-aged population to fit the spectrum of this galaxy (Fig. 14e). The HOBL can be identified. There is some contamination of the $\mathrm{Ca}$ II $\mathrm{H}+\mathrm{H} \epsilon$ absorption by $\mathrm{Ne}$ III $23968+\mathrm{H} \epsilon$ emission.

\subsubsection{Fairall 316}

The $W$ values are very similar to those of the old bulge template, and Figure $14 f$ shows that the latter is indeed a good representation of the nuclear spectrum of this galaxy. There is some contamination of the $\mathrm{Ca}$ II $\mathrm{H}+\mathrm{H} \epsilon$ absorption by $\mathrm{Ne}$ III $\lambda 3968+\mathrm{H} \epsilon$ emission.

\subsubsection{ESO 417-G6}

The near-UV $W$ values, CR, and overall spectrum of this galaxy are best reproduced by the combination of a bulge template plus $10 \%$ mass contribution of a $1 \mathrm{Gyr}$ stellar population (Fig. 14g). The HOBL are at the detection limit but can be identified because there seems to be no contamination by $\mathrm{H} 9$ and $\mathrm{H} 10$ emission. There is some contamination of the $\mathrm{Ca}$ II $\mathrm{H}+\mathrm{H} \epsilon$ absorption by Ne III $\lambda 3968+\mathrm{H} \epsilon$ emission.

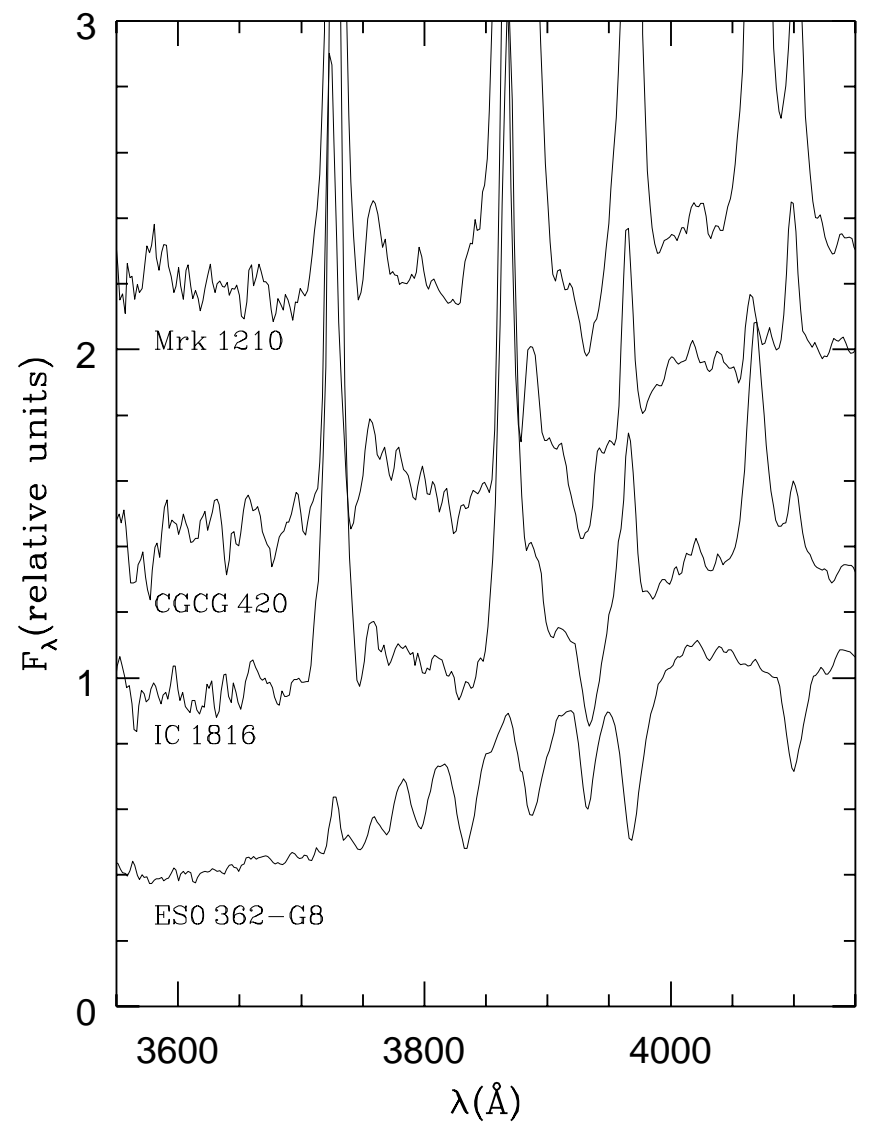

Fig. 10.-Spectra of the four Sa Seyfert type 2 galaxies of the sample normalized at $4020 \AA$ and shifted for clarity.

In summary, of the seven nuclear spectra of S0 Seyfert type 2 galaxies, two can be reproduced by a bulge stellar population and three are better reproduced by a combination of the bulge template with the PL/YS component. The HOBL signatures can be observed in IRAS 11215 - 2806 and ESO 417-G6 because of a large contribution of an intermediate-aged $1 \mathrm{Gyr}$ stellar population.

\section{3. $\mathrm{Sa}$}

\subsubsection{Markarian 1210}

CR is 0.83 for this galaxy, suggesting a PL/YS component contributing with $\approx 50 \%$ of the flux at $4020 \AA$, which is in agreement with the value obtained from $W_{\text {Са п К }}$. The other two $W$ values are contaminated by emission lines, which precludes the detection of the HOBL. Storchi-Bergmann et al. (1998) have shown that the extranuclear spectrum is dominated by an intermediate-aged population, which can be represented by our combination of a bulge plus $1 \%$ mass contribution of a $500 \mathrm{Myr}$ stellar population. They have also shown that the nuclear spectrum could be well reproduced by the latter population plus a very young starburst, as evidenced by the Wolf-Rayet features (Storchi-Bergmann et al. 1998; Cid Fernandes et al. 1999). We have then constructed a model combining $70 \%$ in flux at $4020 \AA$ of the extranuclear population plus $30 \%$ of the $3 \mathrm{Myr}$ population to represent the nuclear spectrum of this galaxy, which is shown together with the observed nuclear spectrum in Figure $15 a$.

The emission lines in the nuclear spectrum are stronger than in the model, indicating that the observations are con- 


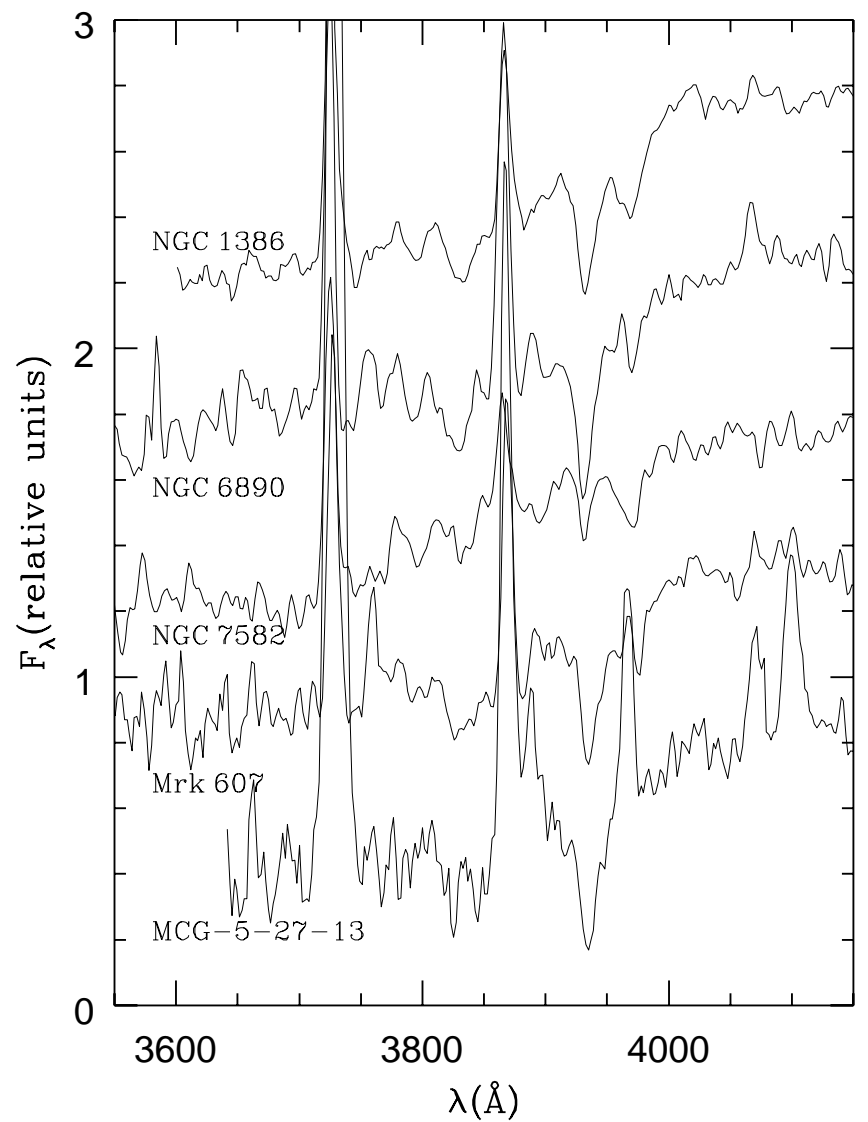

FIG. 11.-Spectra of the five Sb Seyfert type 2 galaxies of the sample normalized at $4020 \AA ̊$ and shifted for clarity.

sistent with the contribution of a 3 Myr stellar population but suggesting also that another source of continuum - the AGN continuum - is necessary to ionize the gas.

\subsubsection{CGCG 420-015}

The near-UV spectrum is best reproduced by a mixture of bulge plus $10 \%$ in mass of an intermediate-aged $(1 \mathrm{Gyr})$ stellar population (Fig. 15b). The spectrum is somewhat noisy in the region, which, together with some contamination by emission lines, makes the identification of the HOBL difficult.

\subsubsection{IC 1816}

The CR of 0.66 suggests $\approx 15 \%$ contribution of a PL/YS continuum. The $W$ values suggest additional dilution, which can be provided by an intermediate-aged population. We thus show in Figure $15 c$ two models: the bulge plus $15 \%$ flux contribution at $4020 \AA$ of the PL/YS component and the improved fit provided by combining the bulge with $10 \%$ in mass of a $1 \mathrm{Gyr}$ stellar population before the combination with the PL/YS. The strong emission precludes a firm identification of HOBL.

\subsubsection{ESO 362-G8}

The nuclear spectrum of this galaxy unambiguously shows all HOBL in absorption. The near-UV $W$ values are well reproduced by the bulge template combined with $10 \%$ mass contribution of a stellar population with age between 100 and $500 \mathrm{Myr}$, with the metal lines of the Seyfert 2 galaxy somewhat deeper, most probably because of the lower

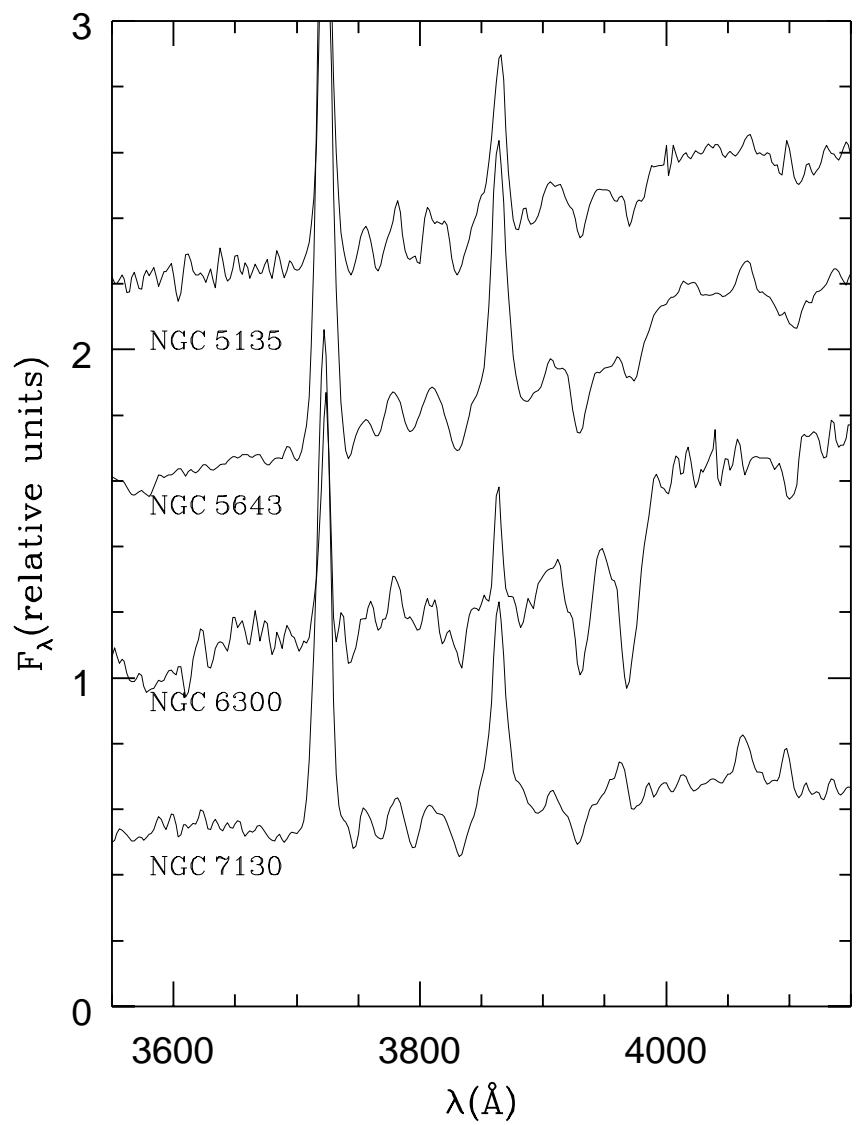

Fig. 12.-Spectra of the four Sc Seyfert type 2 galaxies of the sample normalized at $4020 \AA ̊$ and shifted for clarity.

metallicity of the star clusters used to construct the young stellar templates. The CR measured for this galaxy was 0.35 , much lower than the lowest template value $(0.55)$, suggesting that the continuum was still reddened. We have thus corrected the continuum of this galaxy by an additional $E(B-V)=1$, which brought the CR value to 0.52 (about the minimum obtained for the sample), in agreement with the model selected from the $W$ values (Fig. 15d). This very high reddening is consistent with the dust lane observed crossing the nuclear region (e.g., Malkan et al. 1998) and is in agreement with the $E(B-V)$ values obtained from emission lines (Fraquelli, Storchi-Bergmann, \& Binette 2000).

In summary, among the four Sa galaxies, there is one unambiguous case of a relatively evolved nuclear starburst, ESO 362-G8, clearly showing the HOBL in absorption. In addition, Mrk 1210 has a very young burst, for which the HOBL are filled with emission. For CGCG 420-015 and IC 1816 , there is some contribution of an intermediate-aged population and, for the latter, there is also evidence of a $\mathrm{PL} / \mathrm{YS}$ continuum.

\section{4. $S a b, S b$, and $S b c$ \\ 4.4.1. $N G C 1386$}

The $W$ values, CR, and spectrum are best reproduced by the bulge template plus $10 \%$ in mass of a $1 \mathrm{Gyr}$ population. This fit is shown in Figure 16a, where the HOBL can be observed. The Ne III $\lambda 3968+\mathrm{H} \epsilon$ emission are almost filling the $\mathrm{Ca}$ II $\mathrm{H}+\mathrm{H} \epsilon$ absorption. 

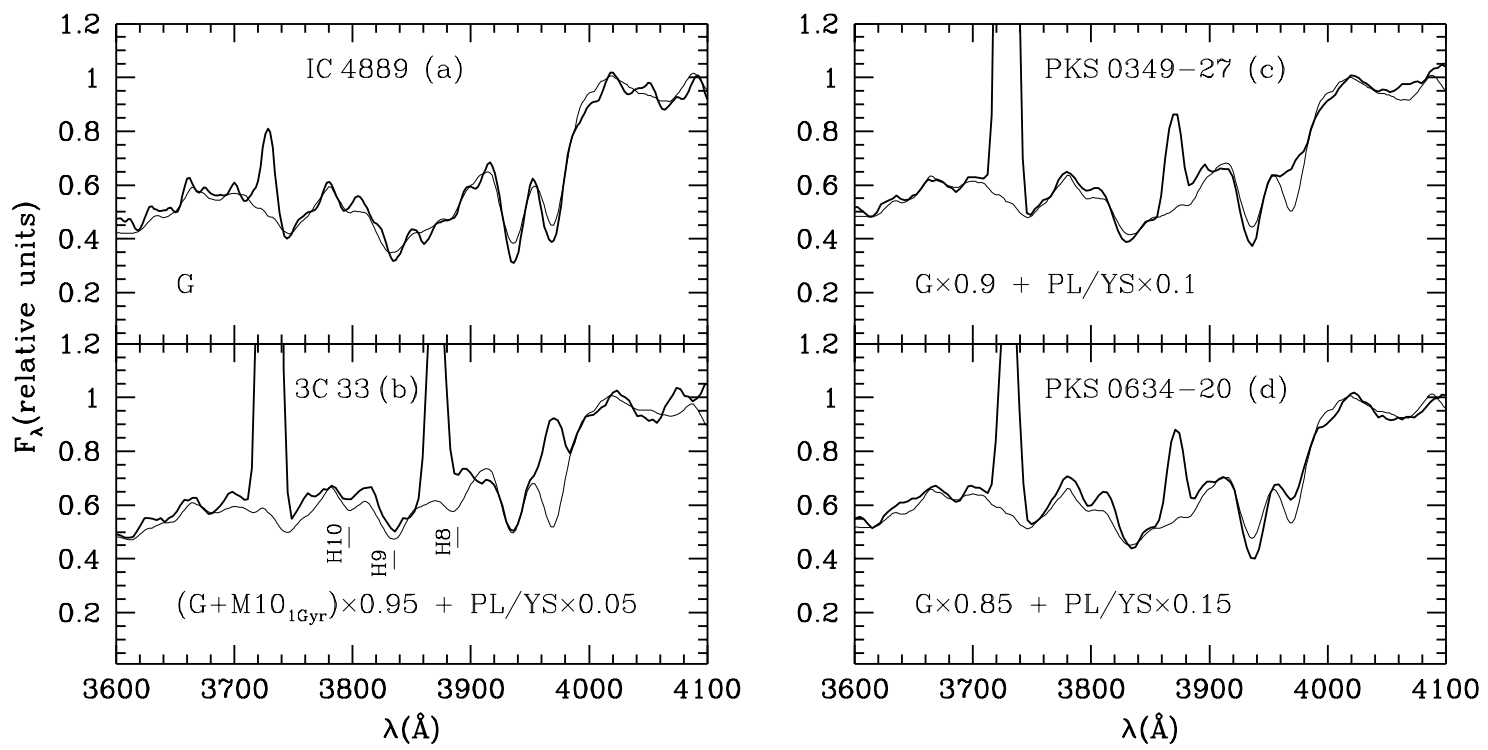

FIG. 13.-Comparison of the nuclear spectrum of the normal elliptical galaxy IC 4889 and the three radio ellipticals (heavy lines) with the best models (thin lines). Labels: $\mathrm{G}$ represents the bulge template, $\mathrm{M} x_{y}$ represents a burst of age $y$ contributing with $x \%$ (in mass) of the bulge mass and PL/YS is the power-law/young stars component. High-order Balmer lines H10, H9, and H8 (HOBL) are identified by vertical lines.

\subsection{2. $N G C 6890$}

The CR of 0.7 indicates the presence of a PL/YS continuum contributing with $\approx 30 \%$ to the flux at $4020 \AA$, in agreement also with the $W$ values. Some contribution of an intermediate-aged population is also possible, and a model including $10 \%$ mass contribution of a $1 \mathrm{Gyr}$ stellar population, combined with $20 \%$ in flux of a PL gives a slightly improved fit in the H9-H10 region, as shown in Figure $16 b$. $\mathrm{Ne}$ III $\lambda 3968+\mathrm{H} \epsilon$ emission are filling the $\mathrm{Ca}$ II $\mathrm{H}+\mathrm{H} \epsilon$ absorption.

\subsubsection{NGC 7582}

This galaxy is well known from the emission lines (diagnostic diagrams) to present a composite Seyfert $2+$ Starburst spectrum. The HOBL are easily seen (Fig. $16 c$ ). The near-UV $W$ values, CR, and spectral distribution are well represented by a bulge template plus $1 \%$ mass contribution of a $50 \mathrm{Myr}$ stellar population. Again, we attribute the poor fit of the $\mathrm{Ca}$ II $\mathrm{K}$ line mostly to the lower metallicity of the young templates as compared to the nuclear region of the galaxy. There is contamination of the $\mathrm{Ca}$ II $\mathrm{H}+\mathrm{H} \epsilon$ absorption by $\mathrm{Ne}$ III $\lambda 3968+\mathrm{H} \epsilon$ emission.

\subsubsection{Markarian 607}

The near-UV $W$ values, CR, and spectrum are best reproduced by a bulge plus $10 \%$ mass contribution of a $1 \mathrm{Gyr}$ stellar population (Fig. 16d). The spectrum is somewhat noisy in the region, and thus the HOBL cannot be unambiguously identified. $\mathrm{Ne}$ III $\lambda 3968+\mathrm{H} \epsilon$ emission are filling the $\mathrm{Ca}$ II $\mathrm{H}+\mathrm{H} \epsilon$ absorption.

$$
\text { 4.4.5. } M C G-05-27-013
$$

CR is 0.7 for this galaxy, suggesting a $20 \%$ contribution of a PL/YS component. The spectrum is dominated by line emission, including in the high-order Balmer lines, and the blue end of the spectrum is noisy. It is not possible to identify the HOBL in absorption (Fig. 16e).

In summary, for the five $\mathrm{Sab}, \mathrm{Sb}$, and Sbc galaxies, NGC 7582 presents the HOBL signatures of a nuclear starburst; NGC 1386, Mrk 607, and possibly also NGC 6890 have
$10 \%$ contribution in mass of intermediate-aged $1 \mathrm{Gyr}$ stars; and the CR of MCG -05-27-013 and NGC 6890 suggest the presence of a PL/YS component contributing with $20 \%$ in flux at $4020 \AA$.

\subsection{Sc and $S d$ \\ 4.5.1. $N G C 5135$}

The HOBL are clear in the spectrum. The $W$ values, CR, and other features of the spectrum can be approximately reproduced by the combination of $50 \%$ each in flux at 4020 $\AA$ of two of our models: the first is the bulge template combined with $1 \%$ in mass of a $10 \mathrm{Myr}$ stellar population, and the second is the bulge template combined with $1 \%$ in mass of a $100 \mathrm{Myr}$ stellar population (Fig. 17a). There is some line emission of $\mathrm{H} \epsilon+\mathrm{Ne}$ III $23968, \mathrm{H} 8$, and possibly H9.

The starburst in this galaxy has been studied extensively by González Delgado et al. (1998) and González Delgado, Heckman, \& Leitherer (2001), hereafter GD98 and GD01. GD98 have shown that the UV spectrum presents clear signatures of $\mathrm{O}$ and $\mathrm{B}$ stars, estimating an age between 3 and $5 \mathrm{Myr}$ for the burst, while GD01 concludes that there is also a similar contribution in flux from an intermediateaged population and a small contribution of an old component. Our model is consistent with the latter mixture.

\subsection{2. $N G C 5643$}

The near-UV $W$ values, CR, and spectrum can be best reproduced by the combination of the bulge template plus $1 \%$ in mass of a $100 \mathrm{Myr}$ stellar population, except for the $\mathrm{Ca}$ II $\mathrm{K}$ line, mostly because of the lower metallicity of the young template, as discussed previously. The HOBL can be identified (Fig. 17b). Ne III $\lambda 3968+\mathrm{H} \epsilon$ emission are almost filling the $\mathrm{Ca}$ II $\mathrm{H}+\mathrm{H} \epsilon$ absorption.

\subsection{3. $N G C 6300$}

The near-UV $W$ values, CR, and spectral distribution are best reproduced by a mixture of a bulge template plus $10 \%$ mass contribution of a $1 \mathrm{Gyr}$ stellar population. The model 

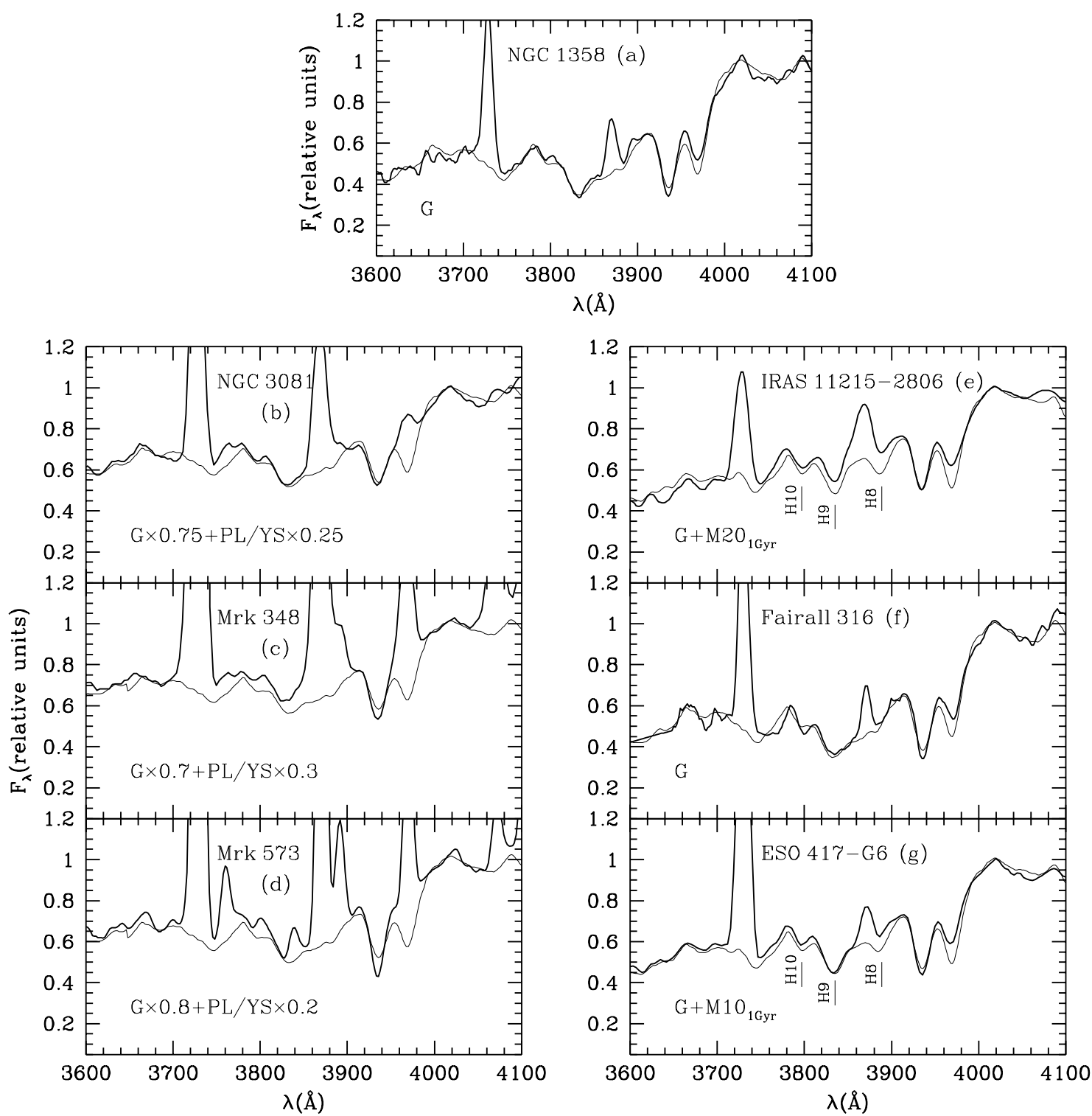

Fig. 14.-Comparison between the nuclear spectrum of the S0 Seyfert galaxies (heavy lines) and the best models (thin lines). Labels as in Fig. 13. Models for Mrk 348 and Mrk 573 include the Balmer continuum from the emitting gas.

is compared with the data in Fig. 17c, where the HOBL are at the limit of detection.

\subsection{4. $N G C 7130$}

Figure $17 d$ shows the clear HOBL in this spectrum, another case of nuclear starburst well studied by GD98 and GD01. The latter authors have suggested the same population as that derived for NGC 5135. Although the $W$ values support the same population, the bluer continuum of NGC 7130 suggests a somewhat younger population, or a mixture including a larger proportion of the younger components. The model we show in the figure is the same composite model as the one adopted for NGC 5135, with a somewhat larger contribution in flux at $4020 \AA$ of the bulge combined with the $10 \mathrm{Myr}$ stellar population: $75 \%$, while the bulge combined with the 100 Myr population contributes with $25 \%$ of the flux.

In summary, for the four Sc and Sd Seyfert galaxies, three present recent episodes of star formation in the nuclear region, with the HOBL clearly visible in the spectra, while one presents $\approx 10 \%$ mass contribution of an intermediateaged population.

\section{COMPARISON WITH OTHER WORKS}

The above results can be compared with those from the synthesis of SSC99. There is good agreement for most galaxies for which the HOBL are observed in the spectra: the young components in our simple models coincide, approximately, with the dominant young components obtained by SSC99. One systematic difference seems to be the contribution of the intermediate-aged components, found to be present in most cases by SSC99, but only in approximately half of the sample here. We attribute this difference to two factors: (1) the models of SSC99 were not as strongly constrained in the blue end of the spectrum as in the present work, providing an optimized representation of the data over the spectral range $\lambda \lambda 3700-7000$; (2) the lower metallicity of some of the star clusters used to construct the $1 \mathrm{Gyr}$ template, which is a mixture of spectra of LMC and Milky Way disk clusters, while the synthesis performed by SSC99 

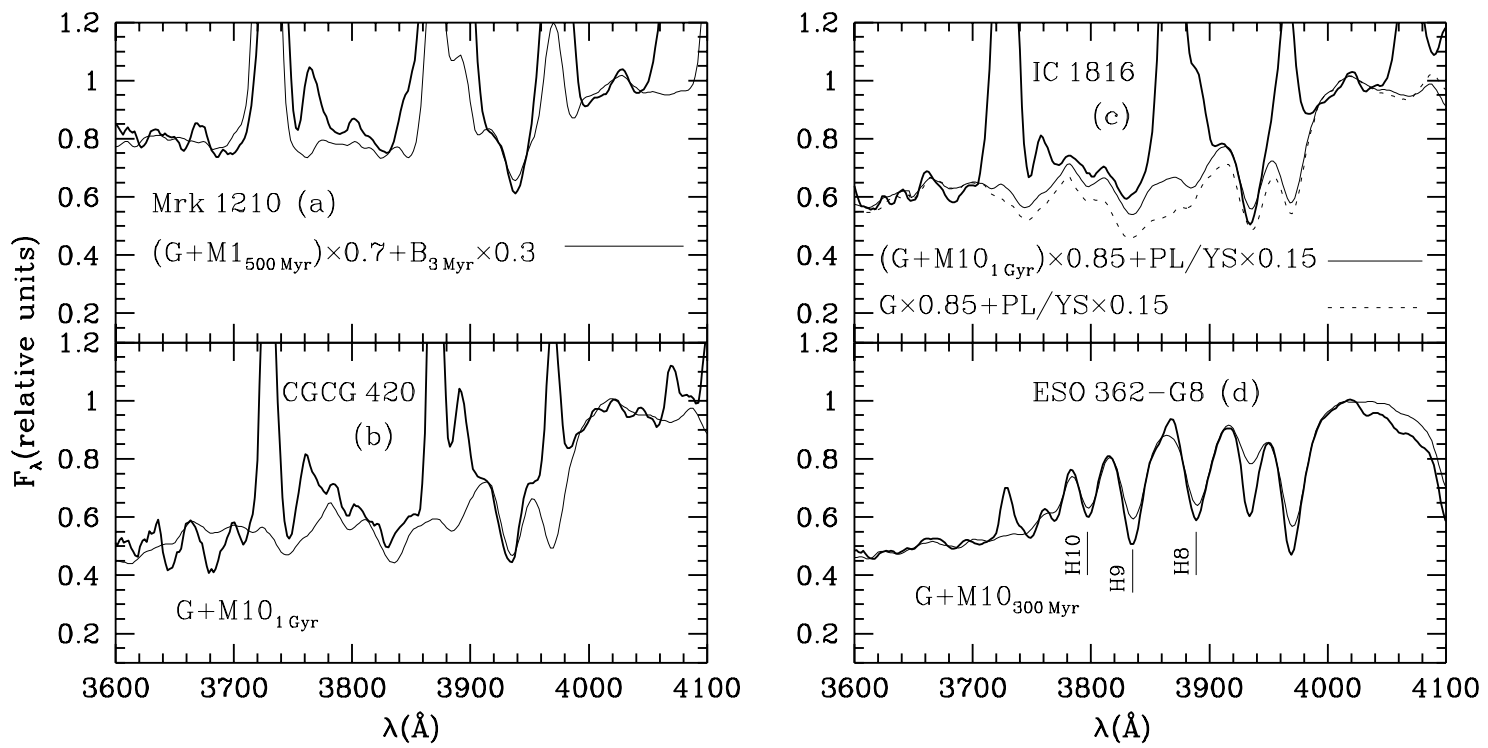

FIG. 15.-Comparison between the nuclear spectrum of the Sa Seyfert galaxies (heavy lines) and the best models (thin lines). Most labels as in Fig. 13 with $\mathrm{B}_{3 \mathrm{Myr}}$ representing the $3 \mathrm{Myr}$ burst. Models for Mrk 1210 and IC 1816 include the Balmer continuum from the emitting gas.

is based on a grid of parameters including the high metallicity end.

GD01 recently have finished a similar spectral study in which they investigate the age of the stellar population at and around the nucleus of a sample of (also) 20 Seyfert type 2 galaxies. They cover the spectral region $\lambda \lambda 3700-4400$ at a similar spectral resolution to our observed spectra but use models with the same resolution. This better resolution in
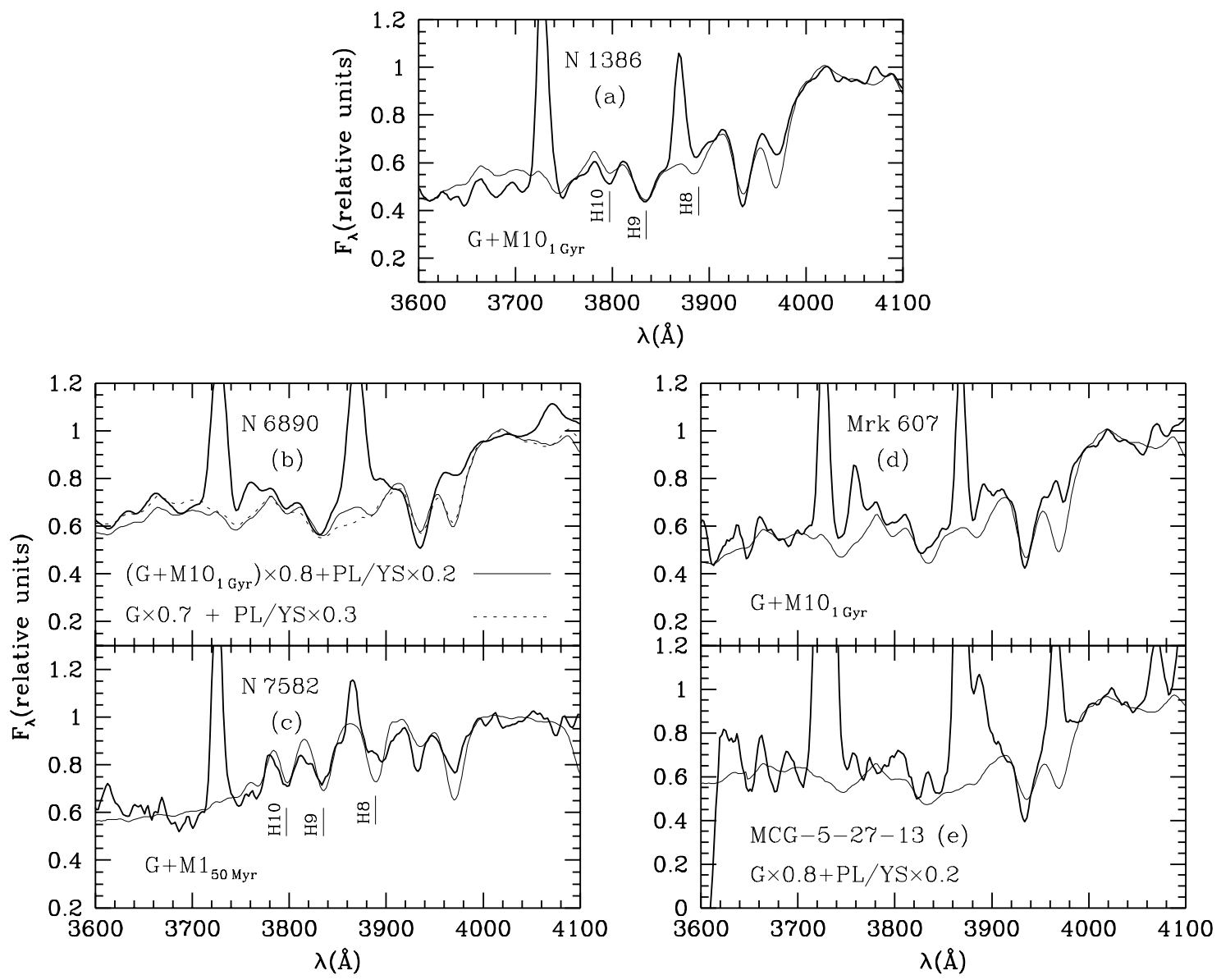

FIG. 16. - Comparison between the nuclear spectrum of the Sb Seyfert galaxies (heavy lines) and the best models (thin lines). Labels as in Fig. 13. Model for MCG - 05-27-13 includes the Balmer continuum from the emitting gas. 

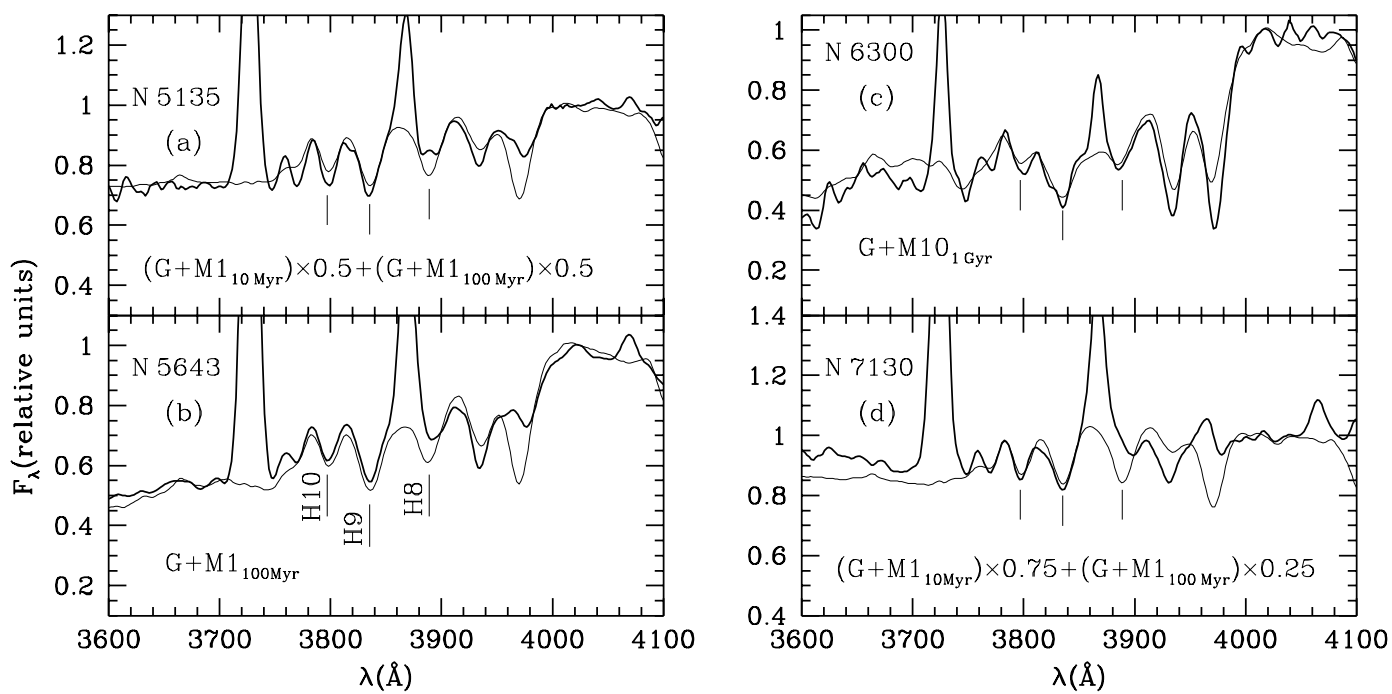

Fig. 17.-Comparison between the nuclear spectrum of the Sc Seyfert galaxies (heavy lines) and the best models (thin lines). Labels as in Fig. 13.

the modeling allows the detection of the He I absorption (e.g., $\lambda \lambda 3819,4387$, and 4922) in some cases, providing a better dating of the starbursts. The higher spectral resolution also allows a more precise evaluation of the emission-line contamination in the high-order Balmer lines.

GD01 find signatures of recent star formation in the nuclear spectrum of six galaxies plus three or four cases in which these signatures are found once the nebular Balmer emission lines are subtracted. In two other cases, they find significant contribution of intermediate-aged stars. There are five galaxies in the sample of GD01 in common with the present study: Mrk 348, Mrk 573, NGC 1386, NGC 5135, and NGC 7130. Similar to what we have found, they conclude that Mrk 348, Mrk 573, and NGC 1386 have a dominant old stellar population and that the near-UV nuclear spectrum of NGC 5135 and NGC 7130 is dominated by light from young and intermediate-aged stars.

\section{THE NATURE OF THE PL/YS CONTINUUM}

As pointed out above, there is a degeneracy between the power-law and starburst continua for ages $\leq 10 \mathrm{Myr}$. This occurs for mass contributions of the young component much smaller than those in the models of Figure 6: for example, for a typical $30 \%$ flux contribution of PL/YS at $4020 \AA$, if it is caused by the continuum of a $10 \mathrm{Myr}$ stellar population, the corresponding mass contribution is only $\approx 0.015 \%$ that of the bulge. This degeneracy is illustrated in Figure 18, where we plot the combined spectrum of a bulge and a PL contributing with $20 \%$ and $40 \%$ in flux at $4020 \AA$, together with the combined spectra of the bulge and a 10 Myr star cluster template, for the same proportions in flux as the PL, and with the combined spectrum of the bulge and a 3 Myr star cluster contributing in flux with half the proportions above, namely $10 \%$ and $20 \%$. A young starburst in nature will most probably present a spread in age, and the spectral distribution will probably be more similar to a combination of the 3 and $10 \mathrm{Myr}$ templates.

It can be observed that, for $\lambda \geq 3500 \AA$, the combination with a PL can hardly be distinguished from that with the young burst templates if their contribution to the flux is $20 \%$. The only constraint here could be the strength of the emission lines.
Figure 18 shows that contributions in flux $\geq 40 \%$ at 4020 $\AA$ and good $\mathrm{S} / \mathrm{N}$ spectra are necessary to allow the distinction between a featureless PL and a young starburst in terms of continuum features, such as the HOBL. Additional constraints could be the emission-line strengths and the UV spectrum slope. The UV spectrum can be observed rising

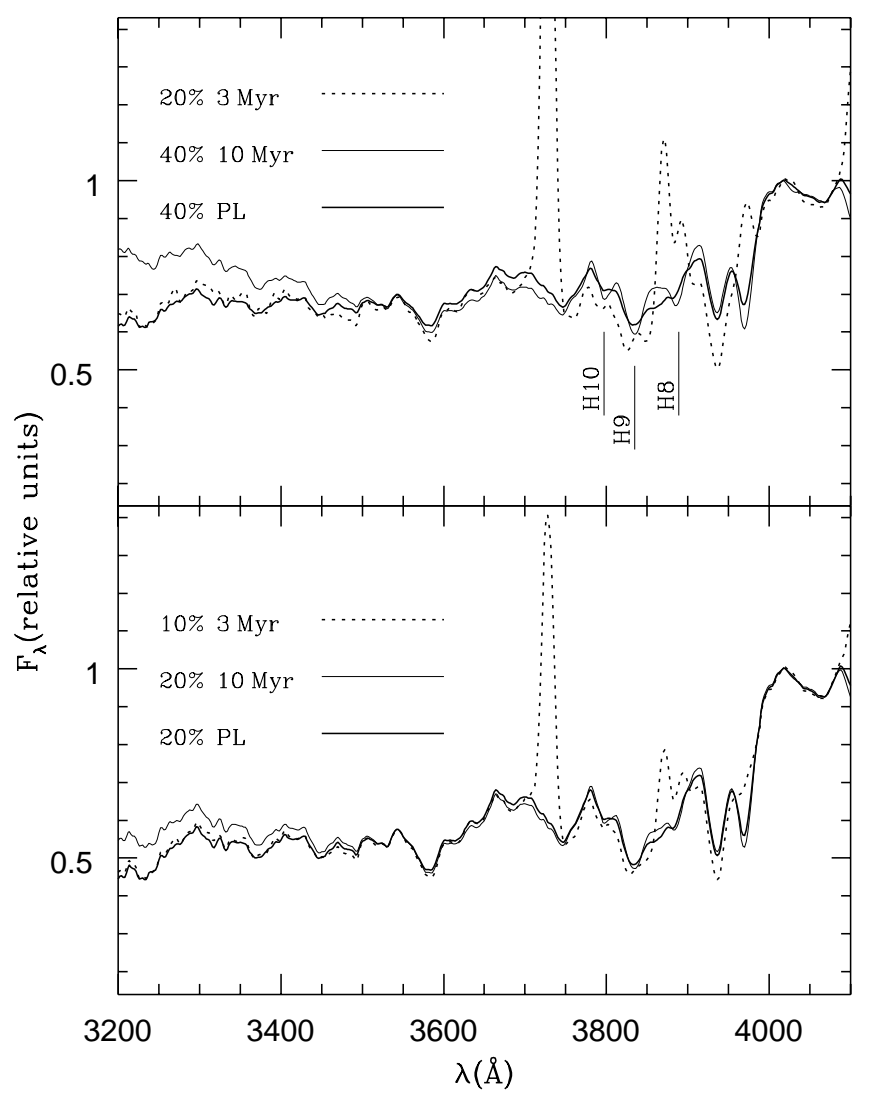

FIG. 18.-Comparison between model spectra constructed combining the bulge template with (1) a PL (heavy line) contributing with $20 \%$ (bottom panel) and $40 \%$ (upper panel) of the flux at $4020 \AA$; (2) a 10 Myr population template (thin line) with the same contribution as the PL; and (3) a $3 \mathrm{Myr}$ population template (dotted line) contributing with $10 \%$ (bottom panel) and $20 \%$ (upper panel) of the flux at $4020 \AA$. 
much more steeply for the $10 \mathrm{Myr}$ stellar population than for the PL. Unfortunately, our data do not extend enough to the UV to allow the use of this constraint.

In our sample, the PL/YS contribution is always smaller than or equal to $30 \%$, and it is thus not possible to determine its origin from the near-UV features alone. In StorchiBergmann et al. (1998), we have discussed the origin of the near-UV continuum of Mrk 348 and Mrk 573: in the former, from the work of Tran (1995a, 1995b, 1995c), $\approx 10 \%$ of the flux comes from scattered light, and thus the remainder could come from young stars. Population synthesis has indeed shown that a mixture of a $10 \mathrm{Myr}$ and a $3 \mathrm{Myr}$ stellar population can reproduce the continuum. In Mrk 573 , at least part of the blue continuum comes from scattered light, as revealed by the images of Pogge \& De Robertis (1993), but we have no constraint on its value.

The only constraint we could try to use here is the strength of the emission lines; for example, if the near-UV continuum were caused entirely by a very young starburst (the $3 \mathrm{Myr}$ one), the equivalent widths $W_{\mathrm{em}}$ values of the Balmer emission lines in the nuclear spectra should be similar to that of the model combining the bulge and the 3 Myr template. Most frequent in nature, however, is the case in which there is some spread in age for the starburst so that the blue continuum is also caused by nonionizing (ageing) blue stars, and the $W_{\mathrm{em}}$ values should then be smaller than in the simple model with the 3 Myr template.

We have measured the equivalent width $W_{\mathrm{H} \beta}$ of the nuclear $\mathrm{H} \beta$ emission line and found that it was smaller than in the simple model above for the two radio galaxies, PKS 0349-27 and PKS 0634-20, and for NGC 6890, and about the same order for NGC 3081. In all other cases, 3C 33, Mrk 348, Mrk 573, and MCG -05-27-13, the observed nuclear $W_{\mathrm{H} \beta}$ values are larger than in the model. We can thus conclude that, if a starburst is the origin of the blue continuum in the above galaxies, it is an ageing one (age $\geq 10 \mathrm{Myr}$ ) for the first three above and could be a very young $(\approx 3 \mathrm{Myr})$ in the other cases. But we also remark that, although the blue continuum could be caused by young stars, the emission-line ratios in all cases (and even the $W$ values in the last three cases) require additional ionizing sources - in other words, the starburst continuum alone cannot account for the emission-line ratios (and emission-line luminosities in the last three cases).

\section{COMPARISON WITH "NORMAL" GALAXIES OF THE SAME HUBBLE TYPE}

A comparison can be made between the near-UV properties of the Seyfert galaxies above and non-Seyfert galaxies of the same Hubble type. Using the Ca II $\mathrm{K} W$ values from Bica \& Alloin (1987) as representative of a sample of normal galaxies, we obtain the following typical values, calculated as averages from approximately 30 galaxies of each Hubble type: (1) for Sa types, $W_{\text {CатK }}=16.5 \pm 1.6 \AA$; (2) for $\mathrm{Sb}$ types, $W_{\text {CarK }}=13.8 \pm 4.2 \AA$; and (3) for Sc types, $W_{\text {CarK }}=9.9$ $\pm 4.9 \AA$. Note that for later types, the spread of $W$ values increases, simply reflecting the variety of mixtures of old (bulge) stellar population and star-forming events in the central region of these galaxies. For ellipticals and S0 types, values similar to those of $\mathrm{Sa}$ galaxies are obtained.

From the 20 Seyfert galaxies, 11 are early-type galaxies, classified as $\mathrm{S} 0$ or Sa; in NGC 1358 and Fairall $316, W_{\text {Са п } \mathrm{K}}$ is typical of early-type galaxies; in the other nine, the $W$ values are smaller, indicating the need of a blue continuum.
This blue continuum is clearly caused by recent (age $<500$ Myr) enhanced star formation in ESO 362-G8 and in Mrk 1210 and by intermediate-aged stars in IRAS $11215-2806$ and ESO 417-G6. For Mrk 348, Mrk 573, and NGC 3081, a $\mathrm{PL} / \mathrm{YS}$ component is favored. This component is at least partially because of scattered light observed as polarized light in Mrk 348 and Mrk 573, but the unpolarized flux could be originated partially in the continuum of a nuclear starburst younger than $10 \mathrm{Myr}$.

Nine sample galaxies have later Hubble types, from $\mathrm{Sb}$ to Sd. Contrarily to the earlier types, the $W_{\text {Са п } \mathrm{K}}$ values are within the range observed for non-Seyfert galaxies. Four of these galaxies present recent (age $<500 \mathrm{Myr}$ ) episodes of star formation in the nuclei, as revealed by the HOBL in NGC 7582, NGC 5643, NGC 5135, and NGC 7130. NGC 1386, NGC 6300, and Mrk 607 are cases in which there is enhanced contribution of an intermediate-aged population of $\approx 1$ Gyr. MCG -05-27-13 and NGC 6890 are cases in which a PL/YS is necessary.

In summary, recent star formation episodes have been found in the nuclei of two of the 11 early-type Seyfert galaxies and in four of the nine late-type Seyfert galaxies, showing a tendency of these episodes to be found more frequently in late-type Seyfert galaxies.

\section{SUMMARY AND CONCLUSIONS}

We have constructed models combining spectral distributions of a typical bulge plus a young stellar population template in the spectral range $\lambda \lambda 3600-4100$ in order to investigate the detectability of recent star formation episodes in the nuclear region of Seyfert galaxies, which usually have prominent bulges.

The high-order Balmer lines (HOBL) are good indicators of the presence of young to intermediate-aged populations as far as the mass contribution of these young components - as compared with the bulge mass-is larger than $1 \%$ for ages of $\approx 100 \mathrm{Myr}$, or larger than $\approx 0.1 \%$ for ages of $\approx 10 \mathrm{Myr}$. We conclude in addition that the continuum ratio $\mathrm{CR}=\lambda \lambda 3660 / 4020$ is an important discriminator of very young stellar populations, as its value can only be larger than $\mathrm{CR}=0.6$ for models including stellar populations younger than $50 \mathrm{Myr}$. If the flux contribution at $4020 \AA$ of these very young populations is smaller than $40 \%$ (corresponding to $0.02 \%$ of the mass of the bulge for the 10 Myr template), it is not possible to distinguish its spectral signatures (e.g., the HOBL) from a featureless power law. Thus, the problem of degeneracy between the featureless AGN continuum and the continuum of a stellar population of $10 \mathrm{Myr}$ or younger still remains at the above flux levels.

By comparing the nuclear spectrum of a sample of 20 Seyfert type 2 galaxies and three radio galaxies with the models above, signatures of recent to intermediate-aged star formation in the form of high-order Balmer absorption lines (HOBL) have been found in nine Seyfert 2 galaxies. From previous analyses of the emission-line features, enhanced recent star formation has been found also in Mrk 1210. In summary, half of our Seyfert 2 sample show signatures of young to intermediate stars. In six cases $(30 \%$ of the sample), the starburst is younger than $500 \mathrm{Myr}$ : ESO 362-G8, NGC 7582, NGC 5135, NGC 5643, and NGC 7130. In the cases of ESO 417-G6, IRAS 11215-2806, NGC 1386, and NGC 6300, the HOBL are caused by a large contribution of intermediate age $(1 \mathrm{Gyr})$ stars. Intermediate-aged stars seem to contribute also to the 
nuclear spectrum of IC 1816, NGC 6890 , and the radio galaxy $3 \mathrm{C} 33$.

The incidence of recent star formation seems to be related to the Hubble type in our sample of 20 Seyfert $2 \mathrm{~s}$ : signatures from young components with ages $<500 \mathrm{Myr}$ have been found in four of the nine late-type galaxies but only in two of the 11 early-type galaxies. This tendency seems to be present also in the sample of González Delgado et al. (2001).

For the remaining five late-type galaxies, two appear to present a PL continuum (alternatively caused by stellar populations younger than $10 \mathrm{Myr}$ ) and three present an intermediate-aged (1 Gyr) component.

Out of the nine early-type galaxies without recent central star formation, the stellar population is well reproduced by the bulge template in two of them, by the bulge plus $10 \%$ contribution in mass of an intermediate-aged population in other three, while in the remaining four, a power law seems to be necessary. The nature of the latter component requires further investigation because of its degeneracy with very young ( $\leq 10 \mathrm{Myr}$ ) stellar population spectra in the wavelength range investigated here. If such component were caused by very young stars in all cases, then the number of
Seyfert 2 galaxies with significant contribution from young stars to the nuclear spectra would increase to $12(60 \%)$ in our sample of 20 Seyfert 2 s.

It is essential to continue the present investigation along two lines: (1) to assess the statistical significance of our findings observing a larger and well-defined sample of Seyfert type 2 galaxies, together with a comparison sample of normal galaxies of the same Hubble types; (2) to investigate the nature of the power-law continuum. The degeneracy of this continuum with those from very young stellar populations could be raised, in principle, in the UV spectral region.

We thank the referee, Rosa González Delgado, for many suggestions that improved the paper. This work has benefited also from discussions with Roberto Cid Fernandes, Henrique Schmitt, and Tim Heckman. We thank the support from the Brazilian institutions Conselho Nacional de Desenvolvimento Científico e Tecnológico, Fundação Coordenação de Aperfeiçoamento de Pessoal de Nível Superior, and Fundação de Apoioà Pesquisa do Estado do Rio Grande du Sul.
Bica, E., \& Alloin, D. 1986, A\&A, 162, 21 1987, A\&AS, 70, 281

Bica, E., Alloin, D., \& Schmidt, A. 1990, MNRAS, 242, 241 (BAS90)

Bica, E., Alloin, D., \& Schmitt, H. R. 1994, A\&A, 283, 805 (BAS94)

Cid Fernandes, R., Lacerda, R. R., Schmitt, H. R., \& Storchi-Bergmann, T. 1999, in IAU Symp. 193, Wolf-Rayet Phenomena in Massive Stars and Starburst Galaxies, ed. K. A. van der Hucht, G. Koenigsberger \& P. R. J. Enens (San Francisco: ASP), 590

Cid Fernandes, R., Schmitt, H. R., \& Storchi-Bergmann, T. 1998, MNRAS, 297, 579

Cid Fernandes, R., \& Terlevich, R. 1992, in ASP Conf. Proc. 31, Relationships between Active Galactic Nuclei and Starbursts Galaxies, ed. Alexei V. Filippenko (San Francisco: ASP), 241

. 1995, MNRAS, 272, 423

de Vaucouleurs, G., et al. 1991, Third Reference Catalog of Bright Galaxies (New York: Springer) (RC3)

Fraquelli, H. A., Storchi-Bergmann, T., \& Binette, L. 2000, ApJ, 532, 867

González Delgado, R. M., Heckman, T. M., \& Leitherer, C. 2001, ApJ, in press

González Delgado, R. M., Heckman, T., Leitherer, C., Meurer, G., Krolik, J., Wilson, A. S., Kinney, A., \& Koratkar, A. 1998, ApJ, 505, 174

González Delgado, R. M., Leitherer, C., \& Heckman, T. M. 1999, ApJS, 125,489

Heckman, T., et al. 1995, ApJ, 452, 549

\section{REFERENCES}

Heckman, T. M., González Delgado, R., Leitherer, C., Meurer, G. R., Krolik, J., Wilson, A. S., Koratkar, A., \& Kinney, A. 1997, ApJ, 482, 114 Leitherer, C., et al. 1996, PASP, 108, 996

Malkan, M. A., Gorjian, V., \& Tam, R. 1998, ApJS, 117, 25

Norman, C., \& Scoville, N. 1988, ApJ, 332, 124

Osterbrock, D. E. 1989, Astrophysics of Gaseous Nebulae and Active Galactic Nuclei (Mill Valley: University Science)

Perry, J. J., \& Dyson, J. E. 1985, MNRAS, 213, 665

Pogge, R. W., \& De Robertis, M. M. 1993, ApJ, 404, 563

Schmidt, A. A., Alloin, D., \& Bica, E. 1995, MNRAS, 273, 945

. 1995, MNRAS, 273, 945

Schmitt, H. R., Storchi-Bergmann, T., \& Cid Fernandes, R. 1999, MNRAS, 304, 35 (SSC99)

Seaton, M. J. 1979, MNRAS, 187, 73P

Storchi-Bergmann, T., Bica, E., Kinney, A. L., \& Bonatto, C. 1997, MNRAS, 290, 231

Storchi-Bergmann, T., Cid Fernandes, R., \& Schmitt, H. R. 1998, ApJ, 501, 94

Storchi-Bergmann, T., Kinney, A., \& Challis, P. 1995, ApJS, 98, 103

Terlevich, E., Diaz, A. I., \& Terlevich, R. 1990, MNRAS, 242, 271

Tran, H. D. 1995a, ApJ, 440, 565 $1995 \mathrm{~b}$, ApJ, 440, 578

.1995c, ApJ, 440, 597 